\title{
The Double Face of Glioma Exosomal microRNAs: Glioma Eliminates Tumor Suppressor miR-1298-5p via Exosomes to Promote Immunosuppressive Effects of MDSCs
}

\section{Hao Xue}

Shandong University Qilu Hospital

Yanhua Qi

Shandong University Qilu Hospital

Chuandi Jin

Shandong University School of Public Health

\section{Wei Qiu}

Shandong University Qilu Hospital

\section{Rongrong Zhao}

Shandong University Qilu Hospital

\section{Shaobo Wang}

Shandong University Qilu Hospital

\section{Boyan Li}

Shandong University Qilu Hospital

\section{Zongpu Zhang}

Shandong University Qilu Hospital

\section{Qindong Guo}

Shandong University Qilu Hospital

Shouji Zhang

Shandong University Qilu Hospital

\section{Zijie Gao}

Shandong University Qilu Hospital

Shulin Zhao

Shandong University Qilu Hospital

\section{Ziwen Pan}

Shandong University Qilu Hospital

\section{Yang Fan}

Shandong University Qilu Hospital

\section{Zihang chen}

Shandong University Qilu Hospital 


\section{Huizhi Wang}

Shandong University Qilu Hospital

Jianye Xu

Shandong University Qilu Hospital

Lin Deng

Shandong University Qilu Hospital

\section{Shilei Ni}

Shandong University Qilu Hospital

Jian Wang

Shandong University Qilu Hospital

\section{Fuzhong Xue}

Shandong University School of Public Health

Gang Li ( $\nabla$ dr.ligang@sdu.edu.cn )

Shandong University Qilu Hospital

\section{Research}

Keywords: glioma, CSF, exosome, microRNAs, MDSCs, TME

Posted Date: July 20th, 2021

DOI: https://doi.org/10.21203/rs.3.rs-688675/v1

License: (c) (i) This work is licensed under a Creative Commons Attribution 4.0 International License. Read Full License 


\section{Abstract}

Background: Clear evidence shows that tumor could secrete microRNAs (miRNAs) via exosomes to modulate tumor microenvironment (TME). However, the mechanisms sorting specific miRNAs into exosomes are still unclear.

Methods: In order to study the biological function and characterization of exosomal miRNAs, we performed whole-transcriptome sequencing in 59 patients' whole course cerebrospinal fluid (CSF) small extracellular vesicles (sEV) and matched glioma tissue samples.

Results: The results demonstrate that miRNAs could be divided into exosome-enriched miRNAs (ExomiRNAs) and intracellular-retained miRNAs (CLmiRNAs), and exosome-enriched miRNAs generally play a dual role. Among them, miR-1298-5p was enriched in CSF exosomes and suppressed glioma progression in vitro and vivo experiments. Interestingly, exosomal miR-1298-5p could promote Immunosuppressive effects of myeloid-derived suppressor cells (MDSCs) to facilitate glioma. Therefore, we found miR-1298-5p had different effects on glioma cells and MDSCs. Mechanically, downstream signaling pathway analyses showed that miR-1298-5p plays distinct roles in glioma cells and MDSCs via targeting SETD7 and MSH2, respectively. Moreover, reverse verification was performed on the intracellular-retained miRNA miR-9-5p.

Conclusions: Thus, we hypothesized that tumor-suppressive miRNAs in glioma cells could be eliminated through exosomes and target tumor-associated immune cells to induce tumor-promoting phenotypes. Glioma could get double benefit from it. These findings uncover the mechanisms that glioma selectively sorts miRNAs into exosomes and modulates tumor immunity.

\section{Background}

Glioma is most common primary CNS tumor and has a very poor prognosis, with an average median survival of approximately $14-17$ months $(1,2)$. The malignancy of tumors are not only determined by cancer cells themselves but also depend on the cross-talk between the tumors and their microenvironment (3). TME consists of many different types of immune cells, including tumor-associated macrophages and microglia (TAMs), dendritic cells (DCs), myeloid-derived suppressor cells (MDSCs), neutrophils, lymphoid cells (3-5). Exosomes play an essential role in mediating intercellular communication between tumors and TME (6). Exosomes are 30-150nm nanovesicles, which originate from the endosome and contain nucleic acids, lipids, and proteins. Extensive evidence from recent studies showed that miRNAs in exosomes are important for tumor diagnosis and treatment (7). Exosomal miRNAs could modulate tumor proliferation, migration, angiogenesis, the epithelialmesenchymal transition (EMT) as tumor suppressors or promoters. Moreover, exosomal miRNAs could even affect the TME, influencing the immune cells activation and recruitment (8).

Previous studies demonstrated that miRNAs could be specifically sorted into exosomes via interaction with RNA-binding proteins (9-11). Exosomal miRNAs can be classified as exosome-enriched miRNAs and 
intracellular-retained miRNAs $(12,13)$. Some studies indicated that tumors of the digestive system excreted tumor suppressor miRNAs in exosomes from the body through digestive tract (14). However, for brain tumors and other solid tumors without natural cavities, the fate of these exosomes and the large number of tumor suppressor miRNAs is still unclear.

In our previous study, we found that glioma delivered tumor-promoting miRNAs to immune cells in the TME via exosomes to induce the immune-suppressing type of immune cells, further promoting the tumor progression (15-18). It has become the mainstream view that cancer cells transmit tumor-promoting miRNAs to the immune cells in the TME via exosomes to produce comprehensive tumor-promoting effects. However, it is not completely clear whether tumor suppressor miRNAs in exosomes still play a tumor-suppressive role in the TME.

It is reported that compared with the normal brain, a variety of tumor-suppressive miRNAs of glioblastoma is significantly down-regulated. However, the mechanism is still not clear (19). In the present study, we sequenced the CSF exosomes and matched tumor tissue samples from 59 patients. And we found that miRNAs highly enriched in glioma exosomes were almost tumor-suppressive miRNAs. Some tumor-suppressive miRNAs are always sorted explicitly into exosomes to downregulate their expression level, and some tumor-promoting miRNAs were almost stuck in tumor cells. Thus, they jointly maintain the cancer-promoting signature of miRNAs components in tumor cells. We conducted research on representative tumor-suppressive EXOmiRNA miR-1298-5p and tumor-promoting CLmiRNA miR-9-5p. Results showed that EXOmiRNA miR-1298-5p could inhibit the proliferation of glioma and targeted SETD7 to affect the AKT pathway. Glioma sorted oncosuppressor miR-1298-5p into exosomes via heterogeneous nuclear ribonucleoprotein A2B1 (hnRNPA2B1). On the contrary, CLmiRNA miR-9-5p could promote the progression of glioma and be stuck inside cells.

More importantly, we have also demonstrated for the first time that these tumor-suppressive EXOmiRNAs can be taken up by immune cells in the TME and turn into cancer promoters. In our previous study, we found exosomal miRNAs could target tumor-associated immune cells to modulate tumor immunity (15, $17,18,20)$. Therefore, we overexpressed miR-1298-5p in the macrophage and MDSCs and found miR1298-5p could induce immunosuppressive effects of MDSCs. Furthermore,we revealed miR-1298-5p targeted MSH2 in MDSCs to affect the NF-kB pathway. Thus, we demonstrated that tumor-suppressive miRNAs in glioma cells could be eliminated through exosomes and target tumor-associated immune cells to induce tumor-promoting phenotypes.

Our research has shown that miRNAs have different effects on various cell types (21). We hypothesized that glioma can selectively excrete tumor-suppressive miRNAs from tumor cells via exosomes and deliver them to immune cells in TME to transform them into cancer-promoting phenotypes. This mechanism that tumor cells selectively sort tumor-suppressing miRNAs into exosomes to get double benefit played an important role in tumor progression, which deserved our further attention.

\section{Materials And Methods}


Patients and data collection.

Our study included CSF and tumor samples from 44 patients who were treated for glioma at Qilu Hospital of Shandong University from November 2017 to October 2019. The whole course CSF sEV samples collected preoperatively were defined as 'pre', and the postoperative CSF samples were defined as ' $p 1$ ' to ' $p 6$ ' according to the collecting series. The 12 cases of non-tumorous brain tissues were obtained from the cortex of decompressive surgery patients with brain trauma or hypertensive intracerebral hemorrhage between November 2018 and April 2019 from the Department of Neurosurgery of the Qilu Hospital of Shandong University, the Second Hospital of Shandong University, and the 5th People's Hospital of Jinan Shandong University. And 3 normal CSF (nor) were obtained from shunt procedures of normal pressure hydrocephalus (NPH) patients between February 2018 and December 2018 from the Department of Neurosurgery of the Qilu Hospital of Shandong University. All glioma patients had received surgical treatment microscopically. All patients authorized the informed consent, and this study was conducted in accordance with institutional ethical standards, the Declaration of Helsinki, and national and international guidelines. Ethics approval was obtained from the Clinical Research Ethics Committee of Qilu Hospital Shandong University.

Magnetic resonance imaging.

All patients underwent brain MRIs as indicated by the standard of care with standard sequences including axial T1-weighted, T2-weighted FLAIR and contrast T1-weighted images. Brain MRIs were reviewed by an experienced neuroradiologist without knowledge of the CSF SEV sequencing results. The tumor regions were semi-automatically segmented slice by slice using 3D Slicer (www.slicer.org) on FLAIR and contrast T1-weighted sequence. To truncate outlier intensities, bias correction was applied on FLAIR and contrast T1-weighted sequence images to compensate for intensity non-uniformities using N4 algorithm (22) implemented in "extrantsr" package and then the intensities of images were normalized using "WhiteStripe" package in $\mathrm{R}$ which conducts white stripe normalization procedure (23). Tumor regions registration was performed by using "lesymap" package. The anatomical image of the patient was registered to the same geometric space as the template, the ICBM 152 2009c Nonlinear atlas, with the tumor regions being excluded. The transformation matrices obtained from the above registration were applied to the tumor region, and the tumor region was brought in template space. The tumor burden (sum of the products of the diameters, SPD), radiographic progression and presence or absence of radiographic signs of tumor spread to subependymal, pial and leptomeningeal sites (CSF type) was according to the previous publications (24).

Isolation and characterization of sEV from CSF.

SEVs were isolated from CSF by differential centrifugation, according to the previous publications (25). After removing cells and other debris by centrifugation at $2000 \mathrm{~g}$ for $30 \mathrm{~min}$, the supernatant was centrifuged at $12,000 \mathrm{~g}$ for 45 min to remove shedding vesicles and the other vesicles with bigger sizes. The supernatant was centrifuged at $110,000 \mathrm{~g}$ for $70 \mathrm{~min}$ and re-suspended in $10 \mathrm{~mL}$ PBS. Finally, the suspension was re-centrifuged at $110,000 \mathrm{~g}$ for $70 \mathrm{~min}$ (all steps were performed at $4 \mathrm{C}$ ); sEV were 
collected and re-suspended in 50 $\mathrm{L}$ PBS for further trans-omics RNA sequencing. The transmission electron microscopy assay of CSF sEV pellet was examined and photographed with an FEI Tecnai spirit TEM T12(FEI Tecnai Spirit 120 kv), according to the previous publications (25).

RNA library preparation and sequencing.

Total RNA from tissues and sEV of CSFs was isolated by using TRIzol Reagent (Invitrogen) according to the manufacturer's instructions. RNA quality and quantity were assessed using a Nanodrop 2000 spectrophotometer (Thermo Fisher Scientific) and Agilent 2100 bioanalyzer (Agilent Technologies). Shortchain RNAs (miRNAs) and long-chain RNAs (mRNAs, IncRNAs and circRNAs) libraries were prepared by using NEBNext ${ }^{\circledR}$ Multiplex Small RNA Library Prep Set for Illumina ${ }^{\circledR}$ (NEB) and NEBNext ${ }^{\circledR}$ UltraTM RNA Library Prep Kit (NEB), respectively. Long-chain RNA and miRNA sequencing was separately performed using the HiSeqX and HiSeq2500 platform (Illumina) according to the Illumina standard protocol by Beijing Novel Bioinformatics Co., Ltd. (https://en.novogene.com/).

Quantification of transcripts abundance.

Clean reads were obtained after removal of reads containing adapters, reads containing ploy- $\mathrm{N}$ and lowquality reads from the raw Illumina sequencing reads. For long-chain RNAs, human reference genome and annotation files were downloaded from the genome website (NCBI/UCSC/Ensembl). Then, clean reads were aligned against the reference genome using HISAT2. To quantify the gene expression level, HTSeq was used to count the read numbers mapped for each gene. For miRNAs, clean reads were aligned against the human reference database (miRbase, http://www.mirbase.org/) using Bowtie and exact matches to known mature miRNA sequences in miRBase were counted. The Transcripts Per Million (TPM) of each miRNA was calculated based on the miRNA read counts. CIRI2 and Find_circ, the circRNA identification algorithms, were employed to predict circRNAs.

Differential expression analysis.

Normalization and differential expression analysis between glioma and control tissues as well as in sEV between preoperative (pre) and control CSF ( $\mathrm{p} 1$ and nor) were performed by using the DEseq2 R package. The P-value was calculated and corrected for multiple testing using the Benjamini-Hochberg method. Normalized expression boxplots and volcano plots were generated by ggplot2 and ggpubr R package. Sample clustering, principal components analysis (PCA), hierarchical clustering were performed by using hcluster, prcomp and pheatmap R package, respectively.

The miRNA target prediction.

Based on the differentially expressed mRNAs, IncRNAs and circRNAs between glioma and control tissues, target mRNA, IncRNA and circRNA of differentially expressed miRNAs in sEV of preoperative CSF (pre) and glioma tissue were obtained by these following steps. The target mRNAs were obtained using Targetscan (http://www.targetscan.org/), miRDB (http://www.mirdb.org/), miRTarBase (http://mirtarbase.mbc.nctu.edu.tw/) which were further filtered by Pearson Correlation Coefficient (PCC) 
analysis between the expression levels of mRNA and miRNA in glioma tissues ( $P C C<-0.3$ and $P<0.05$ ); the target IncRNAs were obtained by DIANA-LncBasev2.0 (http://carolina.imis.athenainnovation.gr/diana_tools/web/index.php?r=Incbasev2/index-predicted) and StarBase 2.0 (http://starbase.sysu.edu.cn/starbase2/index.php); the target circRNAs were obtained by miRDB (http://www.mirdb.org/) and StarBase 2.0 (http://starbase.sysu.edu.cn/starbase2/index.php). Finally, a competitive endogenous RNA (ceRNA) network was constructed by using Cytoscape 3.7.0 software after the PCC analysis of expression levels between IncRNA and mRNA $(P C C>0.3$ and $P<0.05)$ as well as circRNA and $\mathrm{mRNA}(\mathrm{PCC}>0.3$ and $\mathrm{P}<0.05)$ in glioma tissues.

Pathway enrichment analysis.

Pathway enrichment analysis was done on target genes of up- and down-regulated miRNAs in preoperative CSF sEV (pre) compared with glioma tissue using the clusterProfiler R package. Firstly, symbol gene IDs were converted to Entrez gene IDs. Then, Kyoto Encyclopedia of Genes and Genomes (KEGG) enrichment analysis was implemented.

Cell culture.

P3 cell line was kindly provided by Prof. Rolf Bjerkvig, University of Bergen, which was isolated from human glioblastoma tissue. P3 cells were maintained in neurobasal medium (NBM) supplemented with GlutaMAX (2mM), B-27(1x), penicillin/streptomycin (1x), heparin(32 IE/ml), EGF(20 ng/ml) and FGF2(20 $\mathrm{ng} / \mathrm{ml}$ ). U87MG, U251, A172 (Chinese Academy of Sciences Cell Bank) and LN229 cells (ATCC) were cultured in DMEM (Sigma) supplemented with 10\% FBS (Thermo Fisher Scientific). All the cell lines were incubated at $37^{\circ} \mathrm{C}$ with $5 \% \mathrm{CO} 2$ and $95 \%$ air. All cells were authenticated by short tandem repeat (STR) profiling and routinely tested for mycoplasma contamination.

Induction of M2 Macrophages.

PBMCs were isolated from the blood of healthy donors as previously described (15). Blood was separated using standard density gradient centrifugation (30 minutes at $500 \mathrm{~g}$ at $21^{\circ} \mathrm{C}$, LTS1077, TBD). PBMCs were extracted from the interphase. CD14 + cells were selected using magnetic CD14-positive beads (Miltenyi Biotec, 130-050-201). To differentiate these monocytes into monocyte-derived macrophages, CD14 + cells were cultured in RPMI 1640 media (Thermo Fisher Scientific) supplemented with $10 \%$ FBS and 100ng/ml M-CSF (PeproTech). Six days later, the cells were cultured in $0.5 \mathrm{ml}$ OptiMEM containing $4 \mu \mathrm{l}$ Lipofectamine 3000 and $20 \mathrm{pmol}$ mimics. The Opti-MEM was replaced with complete RPMI 1640 medium 6 hours later. Two days later, cells were stained with anti-CD206-APC (Invitrogen, 172069-42). Flow cytometry was performed using the Beckman Coulter Gallios and data was analyzed using FlowJo software.

RNA extraction and quantitative reverse-transcription (qRT-PCR)

Total cell RNA was extracted using RNA-Quick Purification Kit (ESscience Biotech, China) according to the manufacturer's protocol. ReverTra Ace qPCR RT Master Mix (Toyobo, Japan) was used to synthesize 
cDNA following the manufacturer's instruction. qRT-PCR was performed with SYBR Green PCR Master Mix (Applied Biosystems, Foster City, USA). Expression data of microRNA and mRNA were normalized to the internal controls U6 and GAPDH, respectively. The relative expression levels were calculated using the $\Delta \Delta \mathrm{Ct}$ method. The sequence of primers are shown in Supplementary Table 6.

\section{Western blotting}

Whole-cell protein was extracted from glioma cells and MDSCs in RIPA buffer (Thermo Fisher Scientific, USA) and centrifuged at 12,000 rpm for $20 \mathrm{~min}$. A BCA kit (Thermo, Waltham, MA, 23228) was used to measure the protein concentration. After immunoblotting, the proteins were transferred to a nitrocellulose membrane and incubated with specific antibodies. The following primary antibodies were used: $\beta$-actin (Proteintech, 60008-1-Ig), CyclinD1 (Cell Signaling Technology, 2978), P27 (Cell Signaling Technology, 3686), CDK6 (Cell Signaling Technology, 3136), p-AKT (Cell Signaling Technology, 4060), AKT (Cell Signaling Technology, 4691), SETD7 (Cell Signaling Technology, 2813), hnRNPA2/B1 (Cell Signaling Technology, 9304), Phosphorylated NF-kB p65 (S536) (Cell Signaling Technology, 3033), NF-kB p65 (Cell Signaling Technology, 8242).

Small interfering RNA, miR mimics and adenovirus vector transfection

Control microRNAs, miR-1298-5p mimics, were purchased from GenePharma (Shanghai, China). si-setD7, si-MSH2 and control siRNAs were purchased from RiboBio (Guangzhou, China). All sequences are listed in Supplementary Table 6. For microRNA and siRNA transfection, cells were incubated in 6-well plates overnight and transfected with LipofectamineTM 3000 reagent (Thermo Fisher Scientific, USA) according to the manufacturer's protocol. The miR-1298-5p overexpression and control lentiviruses were synthesized by Genechem (Shanghai, China). The knockdown/overexpression efficiency of the siRNAs and virus are available in the supplementary materials.

CCK8 assay

Cell Counting Kit-8 (CCK-8) was used to measure cell viability according to the manufacturer's instructions (Beyotime, China). Cells were seeded at 5,000 cells per well into 96-well plates and cultured at $37^{\circ} \mathrm{C}$ with different treatments. CCK-8 solution $(10 \mu \mathrm{l})$ was added at 24,48 and $72 \mathrm{~h}$. Following incubation for $2 \mathrm{~h}$, the absorbance at $450 \mathrm{~nm}$ (OD450) was measured using a Multimode Plate Reader (PerkinElmer, USA).

Cell cycle analysis

Glioma cells were and stained with Propidium iodide (PI) in the presence of RNase A for 15 min. Flow cytometer (BD Biosciences) was used to perform cell cycle analysis according to the protocol.

Ethynyl-2'-deoxyuridine (EdU) cell proliferation assay 
EdU assay kit (Ribobio, China) was used to test the cell proliferation ability according to the manufacturer's instructions. Glioma cells were seeded into wells of poly-l-ornithine precoated 12-well plates. Cells were then incubated with $200 \mu$ of 5 -ethynyl-20-deoxyuridine for $2 \mathrm{~h}$ at $37^{\circ} \mathrm{C}$. Nuclei were counterstained with Hoechst 33342. Representative images were obtained with a Leica inverted fluorescence microscope.

Dual-luciferase reporter assay

The dual luciferase reporter plasmids (SETD7 WT/MUT and MSH2 WT/MUT) were designed and synthesized by GenePharma (Shanghai, China). HEK-293T cells were seeded in 96-well plates overnight ( $2 \times 104$ / well). For 3'UTR tests, the dual luciferase reporter plasmids $(0.1 \mu \mathrm{g} /$ well) were co-transfected with miR-Nc and miR-1298-5p mimics $(20 \mathrm{nM} \times 0.5 \mu \mathrm{l} /$ well). Approximately 48 hours after transfection, the cells were subjected to luciferase activity analysis using a Dual-Luciferase Reporter Assay System (Promega) following the manufacturer's instructions.

Exosome isolation

Cells were cultured in DMEM supplemented with $10 \%$ exosome-depleted FBS under normoxic $(21 \%$ O2) or hypoxic ( $1 \% 02)$ conditions. Exosomes were isolated from cell culture supernatant as previously described for later analysis.

Electron microscopy and qNano

Isolated exosomes were examined using Transmission Electron Microscopy (TEM) as previously described. qNano (Izon Sciences Ltd, NZ) was used for exosome particle size and concentration analysis. [18]

Cytokine assay

Cell culture medium was collected 72 hours after the indicated treatment. The secretion of TNF- $\beta$ was detected by ELISA (Proteintech, USA) and the NO was measured using Greiss regent, according to the manufacturer's instructions.

RNA Binding Protein Immunoprecipitation

The RIP assays were performed using an EZ-Magna RIP kit (Millipore). Lysates of $1 \times 107$ glioma cells obtained using complete RIP lysis buffer were immunoprecipitated with RIP buffer containing antihnRNPA2B1 antibody-conjugated magnetic beads (Abcam). The precipitated RNAs were analyzed by qRT-PCR. Mouse IgG was used as the negative controls.

RNA pull-down assay

The miR-1298-5p-binding proteins were examined using RNA pull-down assays according to the instructions of the Pierce Magnetic RNA-Protein Pull-Down Kit (ThermoFisher Scientific). Biotinylated 
miR-1298-5p and control sequences were synthesized by GenePharma (Shanghai, China). The cell lysate obtained using a Pierce IP Lysis Buffer (Thermo Fisher Scientific) was incubated overnight with biotinylated miR-1298-5p, followed by precipitation with streptavidin magnetic beads. The retrieved protein was eluted from the RNA-protein complex and analyzed by western blotting.

Animal studies

Luciferase labeled and stably transfected U87MG cells overexpressing miR-1298-5p or vector were injected into the brains of randomly grouped 4-week BALB/c nude mice $(5 \times 105 /$ mouse $)$ to build the orthotopic xenograft model. Bioluminescence imaging was used to image the mouse brains every 5 days after glioma cell implantation. Next, we randomly chose 5 mice in each group and euthanized them on the same day $(10 \mathrm{~d})$. The brains were fixed with paraformaldehyde for further study. The remaining mice (5/group) were kept until death for survival analysis. All procedures that involved mice were approved by and under the requirements of the Animal Care and Use Committee of the Qilu Hospital of Shandong University.

\section{Statistical analysis}

The cut-off value between high and low miR-1298-5p expression was set as the expression level of a median sample. Survival analysis was performed using the Kaplan-Meier method and comparisons were done using the log-rank test. The one-way ANOVA test or Student's t test were used for all other data comparisons using GraphPad Prism 8. All data are presented as the mean \pm standard error and P-values $<0.05$ were considered statistically significant.

\section{Results}

RNA expression pattern of CSF sEV in glioma patients

We performed whole-transcriptome sequencing on CSF sEV collected preoperatively, at 1 week, and every three months postoperatively as well as on the surgical excision samples of 44 glioma patients (Fig. 1a). Together with 15 control, including 12 normal brain tissues and 3 normal CSF, we initially organized a whole-transcriptome sequencing glioma cohort integrated whole-course CSF sEV RNA datasets (Fig. 1b, table. S1). The expression profile of various RNAs (miRNAs, mRNAs, IncRNAs, circRNAs) in CSF was obtained. The miRNAs were abundant in SEV and their expression was stable and had no apparent individual difference (Fig. S1a). In contrast, mRNAs, IncRNAs and circRNAs were low-abundance components in CSF sEV whose expression has significant individual differences (Fig. S1b to d). Unlike the CSF sEV RNA expression pattern, the expression of all these four types of RNAs was abundant and stable in glioma tissues (Fig. S2). The locations of the tumors in all patients were distributed throughout the brain, especially in the convex and parenchyma of the brain. The tumor burden (valuated by SPD) and distance between the tumor and CSF circulation pathway (CSF type) did not affect the expression level of CSF sEV(Fig. 1c, table. S2). 
Our finding showed the mRNAs, IncRNAs and circRNAs in CSF sEV all showed poor ability to indicate tumor-burdened status (Fig. S3a, b, c). These long-chain RNAs had indicative abilities in only tumor tissues and were more suitable for tumor specimen biopsies, not liquid biopsies (Fig. S4). Taken together, only sEV miRNAs could serve as biomarker for indicating tumor-burdened status in CSF liquid biopsy. In addition, the number of days from operation to the time of first post-operative CSF collection should be more than a week to make sure the clearance of pre-operative glioma SEV (Fig. S3d, e).

The miRNAs sEV "trash can"

After differential expression analysis, a total of 443 differentially expressed miRNAs (DEmiRNAs, |log2foldchangel $\geq 1$ and FDR $<0.05$, table. S3) were identified between tumor-burdened CSF and tumor tissues, of which 313 were down-regulated and 130 were up-regulated (Fig. 2a). Then, mRNA targets of down- and up-regulated CSF sEV DEmiRNAs in glioma were obtained (Fig. 2b) and these targets were significantly enriched for many cancer-related pathways (Fig. 2c). A total of 115 high-expressed miRNAs (transcripts per million (TPM) $>100$ in postoperative CSF SEV or glioma tissues) were derived from DEmiRNAs between tumor tissue and CSF sEV. Surprisingly, the majority of these 115 miRNAs were tumor-suppressor miRNAs, although they were up- or down-regulated in tumor-burdened CSF sEV (Fig. 2d, table. S4). Moreover, we preformed differential expression analysis between tumor-burdened CSF and non-tumorous CSF including normal CSF (nor) and No.1 postoperative CSF (p1), of which 167 were down-regulated and 111 were up-regulated (Fig. S5a, table. S5). Then, mRNA targets of down- and upregulated CSF sEV DEmiRNAs in glioma were obtained (Fig. S5b) and these targets were significantly enriched for many cancer-related pathways as above (Fig. S5c).

The top 22 miRNAs (TPM > 10000) accounted for more than $80 \%$ of the total expression,which are mostly present in the list of CSF sEV DEmiRNAs (Fig. 3a). Furthermore, some high-abundant tumor-suppressive miRNAs such as miR-1298-5p, miR-122-5p, miR-204-5p showed absolutely excretion tendency to sEV, and some high-abundance tumor-promoting miRNAs such as miR-9-5p were almost stuck in tumor cells (Fig. 3b).

Therefore, there was no strong evidence to support that the efflux of sEV miRNAs was dependent on random package mechanism on account of the lack of strongly positive correlation miRNAs. And the distribution of miRNAs in tumor cells and sEV might be regulated actively. According to the log2 fold change of miRNAs TPM values in CSF sEV and glioma tissues, we divided the sEV efflux patterns of differential expressed miRNAs into 5 types: high-selective sEV type (HSE, log2 foldchange > 4), lowselective sEV type (LSE, log2 foldchange > 1), mix type (MIX, $1 \geq \log 2$ foldchange $\geq-1$ ), low-selective cell type (LSC,log2 foldchange <-1) and high-selective cell type (HSClog2 foldchange <-4) (Fig. 3c).

Compared with glioma tissues, upregulated DEmiRNAs in CSF sEV were mainly sEV type which enriched more tumor-suppressive miRNAs, and the tumor-promoting miRNAs tended to be cell type, and further evidenced the coincidence that the down-regulated CSF sEV contained more tumor-promoting miRNAs (Fig. 2d).

miR-1298-5p could inhibit the proliferation of glioma in vitro and vivo 
miR-1298-5p was high-selective sEV type according to our classification. In U87MG, U251, LN229, P3 primary GBM cells, the expression level of imR-1298-5p was lower than normal human astrocytes (NHA) (Fig. S6a). In the TCGA and CGGA datasets, the glioma patients with higher miR-1298-5p levels had longer overall survival times (Fig. S6b, S6c).

We overexpressed miR-1298-5p in U87MG, U251, and P3 primary GBM cells. The flow cytometry assay showed that miR-1298-5p could Induce G1/S arrest in glioma (Fig. 4a, 4b). As shown in Fig. 4c, miR-1298$5 p$ overexpression decreased the protein level of proliferation makers. Similarly, Edu assay and CCK-8 assay were performed to examine the inhibitory effects of miR-1298-5p on cell proliferation (Fig. 4d-g).

To determine the function of miR-1298-5p in vivo, we stably overexpressed miR-1298-5p in the luciferaselabeled U87MG cells and injected them into the brains of nude mice (Fig. S6d). Bioluminescent imaging showed that miR-1298-5p-overexpressing glioma cells had lower signals (Fig. 4h, 4i). Additionally, the nude mice implanted with miR-1298-5p-overexpressing glioma cells had a longer survival time (Fig. 4j). Moreover, $\mathrm{HE}$ and $\mathrm{IHC}$ staining proved that miR-1298-5p resulted in a remarkable reduction in tumor growth (Fig. 4k, S6e, S6f). We overexpressed the miR-1298-5p in the U87MG, U251 and P3 cells and found that the protein level of phosphorylated Akt decreased (Fig. 4c).

miR-1298-5p was sorted into exosomes via hnRNPA2B1 and promoted the immunosuppressive effects of MDSCs

Next, we tried to explore whether tumor-suppressive miR-1298-5p has effects on the microenvironment. We overexpressed miR-1298-5p mimics in MDSCs. Interestingly, we found miR-1298-5p promoted the function of MDSCs (Fig. 5c, 5d). We isolated GDEs from the culture medium of glioma cells transfected with miR-1298-5p-overexpressing lentivirus. Transmission electron microscopy (TEM) showed that the exosomes isolated from culture supernatants of the glioma cell lines U87MG and P3 were rounded particles ranged from $30 \mathrm{~nm}$ to $100 \mathrm{~nm}$ (Fig. S7a). Then, qNano analysis determined exosome concentration and size distribution (Fig. S7b). Western blot analysis revealed the presence of TSG101 and the absence of calnexin in GDEs (Fig. S7d). Confocal microscopy showed the internalization of PKH67-labeled glioma exosomes (green) by MDSCs (Fig. S7c). And the GDEs overexpressing miR-1298$5 p$ displayed a stronger MDSC induction ability than the PBS and GDEs (Fig. 5a, 5b). Moreover, the expression of NOS2 and TGF- $\beta$ mRNA was increased after transfected with miR-1298-5p mimics (Fig. 5e, $5 f)$. We next measured the NO and TGF- $\beta$ production in MDSCs and found that NO and TGF- $\beta$ were upregulated in MDSCs supernatants (Fig. $5 \mathrm{~g}, 5 \mathrm{~h}$ ). We cultured MDSCs with T cells to evaluate the T cellsuppressing activity and found that MDSCs transfected with miR-1298-5p were more effective at suppressing $T$ cells than the control group (Fig. $5 \mathrm{i}, 5 \mathrm{j}$ ). Previous studies have demonstrated the classical MDSC activation pathway, which includes AKT-PI3K, NF-KB and STAT3 etc (26). miR-1298-5p upregulated the expression of p-p65 in MDSCs (Fig. 5k).

hnRNPA2B1 has been reported to sort RNAs into exosomes as RBPs selectively (27). Sequence analysis by POSTAR2 indicated a sequence motif and structural preference of the RBP binding site for hnRNPA2B1, which was located in the miR-1298-5p (Fig. 5I) (28). Next, we knocked down hnRNPA2B1 in 
U87MG and P3. And we found that hnRNPA2B1 knockdown reduced the exo/cell ratio of miR-1298-5p (Fig. 5m, 5n). We tested the efficiency of hnRNPA2B1 knockdown (Fig. S7e, S6f). hnRNPA2B1 sorts RNAs into exosomes by recognizing a specific motif (i.e., UUCA). Therefore, we induced mutation at this site of miR-1298-5p. And then, we performed the RNA pull-down assay with biotinylated miR-1298-5p WT and MUT. We found that miR-1298-5p (WT) can bind to hnRNPA2B1 but MUT can't (Fig. 5p). Consistently, RNA immunoprecipitation (RIP) showed enrichment of miR-1298-5p by hnRNPA2B1, validating the interaction between miR-1298-5p and hnRNPA2B1 (Fig. 5o). We transfected glioma cells with Cy3-miR1298-5p and cocultured them with MDSCs. Finally, Cy3-miR-1298-5p was detected in MDSCs, which demonstrated that glioma cells could transfer miR-1298-5p to MDSCs via exosomes (Fig. S7g). Then, we transfected U87MG with Cy3-miR-1298-5p and knocked down hnRNPA2B1 in U87MG and cocultured them with MDSCs. The flow cytometry showed that Internalization of Cy3-labeled miR-1298-5p by MDSCs reduced (Fig. S7h). In addition, we knocked down hnRNPA2B1 in U87MG and P3 and cultured them with MDSCs. The results showed that hnRNPA2B1 knockdown reduced the percentage of CD14+ /HLA-DRlow/- MDSCs population (Fig. 5q-s). Moreover, we detected the expression of miR-1298-5p in MDSCs of patents and found that compared with health donors, miR-1298-5p had higher expression in the patients' samples (Fig. 5t).

Collectively, these findings suggested that miR-1298-5p promoted the immunosuppressive effects of MDSCs via the NF-KB pathway. And hnRNPA2B1 could bind to miR-1298-5p and sorted it into exosomes.

miR-1298-5p targeted setd7 in glioma and MSH2 in MDSCs

We predicted the target genes of miR-1298-5p by Starbase and miRDB (Fig. S6g) $(29,30)$. Next, we studied the interaction between the target genes and glioma proliferation and setd7 was found. After transfection of miR-1298-5p, SETD7 expression was downregulated (Fig. S6h, S6i). We constructed the dual-luciferase reporter plasmids, including the WT and MUT 3'UTR of SETD7 (Fig. 6a). And compared with control miRNA, miR-1298-5p decreased the luciferase activity of the WT plasmid and didn't affect the MUT plasmid (Fig. 6b).

To determine the functional role of SETD7 in the development of the glioma, we used the SETD7 siRNA to perform the knockdown experiment (Fig. S6j, S6k). The results showed that knockdown of SETD7 inhibited the proliferation and the AKT pathway of glioma (Fig. 6e-h). Moreover, our results revealed that the effects of up-regulation of miR-1298-5p on proliferation were attenuated by over-expression of SETD7 (Fig. 7).

To identify the targets of miR-1298-5p in MDSCs, we used the previous result predicted by the online bioinformatic tools. Among them, MSH2 has been reported to be associated with the NF-KB signaling pathway (31). Therefore, MSH2 was hypothesized to be a direct target of miR-1298-5p and responsible for MDSCs activation. The qRT-PCR and western-blot assay were used to determine that MSH2 was downregulated by miR-1298-5p (Fig. S6I, S6m). To confirm the binding site of miR-1298-5p in the 3'UTR of MSH2, we performed a luciferase reporter assay. And miR-1298-5p decreased the luciferase activity of the WT plasmid and didn't affect the MUT plasmid (Fig. 6i, 6j). To explore the function of MSH2 in 
MDSCs, we knocked down MSH2 by using small interfering RNAs in MDSCs (Fig. S6n). The flow cytometry results showed that knocking down MSH2 increased the proportion of CD14 ${ }^{+} \mathrm{HLA}-\mathrm{DR} \mathrm{R}^{\text {low/- }}$ MDSCs population (Fig. 6k, 6l). The qRT-PCR results demonstrated that MSH2 knockdown significantly increased the expression of NOS2 and TGF- $\beta m R N A$ (Fig. S6o, S6p). Next, we measured NO and TGF- $\beta$ production in MDSCs supernatants and got the consistent trend (Fig. 6m, 6n). The T cell-suppressive effect of MDSCs was then detected and MSH2-knockdown MDSCs suppressed T cell proliferation more strongly than the control group (Fig. 6o, 6p). In addition, MSH2 knockdown also affected the NF-kB pathway (Fig. 6q). Moreover, the effects of miR-1298-5p overexpression could be partially attenuated by MSH2 overexpression (Fig. 8).

In conclusion, our results showed that miR-1298-5p targeted setD7 in glioma and MSH2 in MDSCs.

miR-9-5p could promote the progression of glioma and induce the M1 macrophage polarization.

miR-9-5p was reduced in exosomes. Therefore, we hypothesized that miR-9-5p has the opposite effect of miR-1298-5p. To investigate the biological function of miR-9-5p in glioma, we overexpressed miR-9-5p in U87MG, U251. The transwell chamber assays and wound-healing assay showed that miR-9-5p could promote the migratory and invasive ability of glioma cells (Fig. S8a-d). Similarly, EdU assay and CCK-8 assay were performed to examine the effects of miR-1298-5p on cell proliferation (Fig. S8e-g). qRT-PCR was performed to examine the expression of CD163, IL-10, IL-6 and TNF-a. Compared with miR-NC mimics, miR-9-5p could significantly increase the expression of IL-6 and TNF-a, and markedly decrease the expression of CD163 and IL-10 (Fig. S8h).

In conclusion, miR-9-5p could promote the progression of glioma and induce the M1 macrophage polarization.

Immunosuppressive phenotype switch

To investigate the effect of sEV miRNAs in the glioma immune microenvironment, we further transfected the top 22 CSF sEV miRNAs into macrophages and then analyzed the flow cytometry data of immunosuppressive markers CD206. Our data indicated that almost all miRNAs could induce the M2 polarization of macrophages (fig S9). Herein, we believed that glioma cells could modulate the myeloid cells in the tumor microenvironment using sEV tumor-suppressor miRNAs that were not needed by tumor cells to produce a tumor-promoting phenotype transformation.

\section{Discussion}

Accumulating evidence has proved that miRNAs play an essential role in tumor origin and progression (32). The immunosuppressive tumor microenvironment is a complex system closely related to tumor progression (33). And exosomes could transfer miRNAs between glioma and TME. Our previous study found that glioma exosomes could promote glioma-associated macrophage infiltration and M2 polarization and induce the expansion and immunosuppressive function of MDSCs $(14,18)$. In our 
research, we report the different effects of miR-1298-5p on glioma cells and MDSCs, respectively. We performed whole-transcriptome sequencing on CSF exosomes and matched glioma tissues. And we found some high-abundant tumor-suppressive miRNAs such as miR-1298-5p, miR-122-5p, miR-204-5p showed absolutely excretion tendency to SEV and help the tumor to resist antitumor immune. Some highabundant tumor-promoting miRNAs such as miR-9-5p were almost stuck in tumor cells. This finding has not only provided a large number of specific miRNA markers for glioma CSF liquid biopsy but also brought back the view of the classical SEV trash can effect (34).

miR-1298-5p was significantly enriched in CSF exosomes of patients. We demonstrated that miR-1298-5p inhibited the proliferation of glioma targeting SETD7 and glioma sorted it into exosomes via hnRNPA2B1 to promote the glioma progression. miR-1298-5p in exosomes could promote the immunosuppressive ability of MDSCs. Therefore, we show for the first time that miR-1298-5p seems to function differently in glioma cells and MDSCs. And glioma could acquire the double benefit by sorting oncosuppressor miR1298-5p into exosomes.

It has been reported that selective sorting of tumor suppressor miRNA into exosomes promotes tumor progression $(12,14)$. Similarly, we found that miR-1298-5p could target SETD7 to affect AKT pathway to inhibit tumor proliferation. SETD7 (also known as SET7, SET9, or SET7/9) is a methyltransferase for $\mathrm{H} 3 \mathrm{~K} 4$ and belongs to the SET domain-containing proteins, which is related to demethylation of H3K4 (H3K4me2) and induces downstream gene expression. It has been reported that SETD7 plays a critical role in hepatocellular carcinoma, colorectal cancer, lung cancer and so on (35-38). In this study, we found that SETD7 knockdown inhibited the proliferation of glioma via AKT pathway. PI3K-AKT signaling network plays an important role in supporting cancer survival and proliferation in various ways (39). Considering its vital role in glioma proliferation, finding new strategies to deactivate the AKT system is essential to improving clinical outcomes for glioma patients.

Although it has been reported that tumor can sort oncosuppressor miRNA into exosomes to promote tumor progression, the effect of miRNA packaged into exosomes on TME is not clearly. No studies have reported the immunosuppressive effect of miR-1298-5p. We hypothesized that miR-1298-5p in exosomes could resist anti-cancer immune activity. For the first time, we reported that miR-1298-5p targets the MSH2 gene and further potentiates MDSCs by activating the NF-KB pathway. We transfected glioma cells with Cy3-miR-1298-5p and cocultured them with MDSCs. Finally, Cy3-miR-1298-5p was detected in MDSCs, which demonstrated that glioma cells could transfer miR-1298-5p to MDSCs via exosomes. NFKB pathway has been proved to activate MDSCs to resist anti-cancer immune activity (40). We found miR1298-5p activated NF-KB pathway in MDSCs. Therefore, preventing the transport of miR-1298-5p to exosomes is an effective way to weaken the immunosuppressive ability of MDSCs.

Exosomes could regulate the biological functions of recipient cells via miRNAs transfer. The exosomal RNAs are selectively loaded into exosomes by different RBPs (41). It has been reported that hnRNPA2B1 regulates the sorting of miRNA into exosomes by binding to specific motifs (27). In our research, we preformed RIP and RNA pull-down assay and found that hnRNPA2B1 binds to UUCA of miR-1298-5p and 
directs its packaging into exosomes. We knocked down hnRNPA2B1 in glioma cells and found that the the exo/cell ratio of miR-1298-5p decreased. Moreover, we knocked down hnRNPA2B1 in glioma cells and cocultured them with MDSCs. And we found that the proportion of $\mathrm{CD}_{14}{ }^{+} \mathrm{HLA}-\mathrm{DR} \mathrm{R}^{\text {low/- }}$ MDSCs population was decreased. These results may provide unique strategies for eliminating miR-1298-5p in the TME.

On the contrary, miR-9-5p was enriched in tumor tissue. And we showed that miR-9-5p could promote the progression of glioma and be trapped inside cells. Moreover, we also found miR-9-5p could induce M1 polarization, which can inhibit tumor progression. Therefore, glioma leaves miR-9-5p inside cells to promote tumor progression.

Furthermore,we divided the sEV efflux patterns of differential expressed miRNAs into 3 types: sEV type, mix type, cell type. According to their types, different treatment strategies can be adopted. For tumorsuppressive EXOmiRNAs, we could prevent them discharged from tumor cells. For tumor-promoting CLmiRNAs, we could inhibit their downstream signaling pathways or downregulate their expression level.

In summary, our findings reveal that miR-1298-5p plays a different role in glioma cells and MDSCs, respectively. It provided a new mechanism that glioma secreted oncosuppressor miR-1298-5p via exosomes to resist anticancer immune activity to acquire dual benefits. Other tumor-suppressive miRNAs are being researched in our research group, and we got the same result. Moreover, miR-1298-5p was enriched in exosomes of glioma patents, which can be used as a maker to achieve glioma liquid biopsy. In the future, nanoparticle or engineered extracellular vesicles can be used to knock down hnRNPA2B1 targeting glioma cells to block the process. It will provide a theoretical basis for glioma treatment.

\section{Conclusion}

Collectively, our findings demonstrated that glioma secreted oncosuppressor miR-1298-5p via exosomes to promote the Immunosuppressive effects of MDSCs to acquire dual benefits. Thus, we hypothesized that tumor-suppressive miRNAs in glioma cells could be eliminated through exosomes and target tumorassociated immune cells to induce tumor-promoting phenotypes. These findings uncover the mechanisms that glioma selectively sorts miRNAs into exosomes and modulates tumor immunity, which provide a theoretical basis for glioma diagnosis and treatment.

\section{Abbreviations}

CSF: Cerebrospinal fluid, sEV: small extracellular vesicles, BBB: blood-brain barrier, miRNAs: microRNAs, TME: tumor microenvironment, ExomiRNAs: exosome-enriched miRNAs, CLmiRNAs: intracellular-retained miRNAs, MDSCs: myeloid-derived suppressor cells, EMT: epithelial-mesenchymal transition, NPH: normal pressure hydrocephalus, PCA: principal components analysis, PCC: Pearson Correlation Coefficient, NHA: normal human astrocytes, TPM: Transcripts Per Million, TEM: Transmission electron microscopy 


\section{Declarations}

\section{Ethics approval and consent to participate}

All animal experiments were approved by the Institutional Animal Care and Use Committee (IACUC) of Shandong University (Jinan, China).

\section{Consent for publication}

Not applicable.

\section{Availability of data and materials}

The datasets used and/or analyzed during the current study are available in Supplementary Tables and the full original data is available from National Genomics Data Center (GSA: CRA002339) or the corresponding author on reasonable request.

\section{Competing interests}

The authors declare that they have no competing interests.

\section{Funding}

This work was supported by grants from the National Natural Science Foundation of China (Nos.81874083, 82072776, 82072775, 81702468, 81802966, 81902540, 81874082, 81472353), Natural Science Foundation of Shandong Province of China (Nos. ZR2019BH057区 ZR2020QH174), Key clinical Research project of Clinical Research Center of Shandong University (2020SDUCRCA011) and Taishan Pandeng Scholar Program of Shandong Province (No. tspd20210322).

\section{Author Contributions}

H.X., F.Zh.X. and G.L. conceived and designed the study. H.X., W.Q., R.R.Zh., collected and 628assembled the data. H.X., L.D., Sh.L.N. were responsible for provision of the study materials and the patients. H.X., Ch.D.J. analyzed and interpreted the data. H.X., Sh.L.Zh., and Zh.Zh.S. processed the CSF and blood samples. H.X., R.R.Zh., J.W., and X.G.L. provided administrative, material and technical support. Q.D.G., B.Y.L., Y.H.Q., Sh.J.Zh., Y. F., Z.W.P. were responsible for the laboratory research. H.X., Ch.D.J., W.Q., Y.H.Q., R.R.Zh., Q.D.G. and B.Y.L. wrote the 633 manuscript. All authors approved the manuscript.

\section{Acknowledgments}

We thank Institute for Medical Dataology of Shandong University for technical and administrative support of omics dataset construction, the Glioma Big Data Union of Shandong Province (SD-GBDU) for glioma cohort management, the RuWei BioTech Co. Ltd. (Jinan) for CSF liquid biopsy workflow and CSF sEV miRNA profiling report design, the Novogene Co. Ltd and Tianjin Novogene Bioinformatics 
Technology Co., Ltd for sEV Whole-transcriptome Sequencing technical development and support, and the iGeneTech Co. Ltd. (Beijing) for Whole-transcriptome Sequencing support.

\section{References}

1. Reifenberger G, Wirsching HG, Knobbe-Thomsen CB, Weller M. Advances in the molecular genetics of gliomas - implications for classification and therapy. Nat Rev Clin Oncol. 2017,14(7):434-52.

2. Lim M, Xia Y, Bettegowda C, Weller M. Current state of immunotherapy for glioblastoma. Nat Rev Clin Oncol. 2018,15(7):422-42.

3. Galon J, Bruni DJI. Tumor Immunology and Tumor Evolution: Intertwined Histories. 2020,52(1):55-81.

4. Quail D, Joyce JJCc. The Microenvironmental Landscape of Brain Tumors. 2017,31(3):326-41.

5. Sampson J, Gunn M, Fecci P, Ashley DJNrC. Brain immunology and immunotherapy in brain tumours. 2020,20(1):12-25.

6. Hoshino A, Kim HS, Bojmar L, Gyan KE, Cioffi M, Hernandez J, et al. Extracellular Vesicle and Particle Biomarkers Define Multiple Human Cancers. Cell. 2020,182(4):1044-61 e18.

7. An T, Qin S, Xu Y, Tang Y, Huang Y, Situ B, et al. Exosomes serve as tumour markers for personalized diagnostics owing to their important role in cancer metastasis. 2015,4:27522.

8. Su T, Zhang P, Zhao F, Zhang SJFio. Exosomal MicroRNAs Mediating Crosstalk Between Cancer Cells With Cancer-Associated Fibroblasts and Tumor-Associated Macrophages in the Tumor Microenvironment. 2021,11:631703.

9. Hobor F, Dallmann A, Ball N, Cicchini C, Battistelli C, Ogrodowicz R, et al. A cryptic RNA-binding domain mediates Syncrip recognition and exosomal partitioning of miRNA targets. 2018,9(1):831.

10. Lee H, Li C, Zhang Y, Zhang D, Otterbein L, Jin YJTJoem. Caveolin-1 selectively regulates microRNA sorting into microvesicles after noxious stimuli. 2019,216(9):2202-20.

11. Wozniak A, Adams A, King K, Dunn W, Christenson L, Hung W, et al. The RNA binding protein FMR1 controls selective exosomal miRNA cargo loading during inflammation. 2020,219(10).

12. Santangelo L, Giurato G, Cicchini C, Montaldo C, Mancone C, Tarallo R, et al. The RNA-Binding Protein SYNCRIP Is a Component of the Hepatocyte Exosomal Machinery Controlling MicroRNA Sorting. 2016,17(3):799-808.

13. Mills J, Capece M, Cocucci E, Tessari A, Palmieri DJljoms. Cancer-Derived Extracellular VesicleAssociated MicroRNAs in Intercellular Communication: One Cell's Trash Is Another Cell's Treasure. 2019,20(24).

14. Teng Y, Ren Y, Hu X, Mu J, Samykutty A, Zhuang X, et al. MVP-mediated exosomal sorting of miR193a promotes colon cancer progression. Nat Commun. 2017,8:14448.

15. Guo X, Xue H, Shao Q, Wang J, Guo X, Chen X, et al. Hypoxia promotes glioma-associated macrophage infiltration via periostin and subsequent $\mathrm{M} 2$ polarization by upregulating TGF-beta and M-CSFR. 2016,7(49):80521-42. 
16. Guo X, Qiu W, Liu Q, Qian M, Wang S, Zhang Z, et al. Immunosuppressive effects of hypoxia-induced glioma exosomes through myeloid-derived suppressor cells via the miR-10a/Rora and miR-21/Pten Pathways. 2018,37(31):4239-59.

17. Guo X, Qiu W, Wang J, Liu Q, Qian M, Wang S, et al. Glioma exosomes mediate the expansion and function of myeloid-derived suppressor cells through microRNA-29a/Hbp1 and microRNA92a/Prkar1a pathways. 2019,144(12):3111-26.

18. Qian M, Wang S, Guo X, Wang J, Zhang Z, Qiu W, et al. Hypoxic glioma-derived exosomes deliver microRNA-1246 to induce M2 macrophage polarization by targeting TERF2IP via the STAT3 and NFKB pathways. Oncogene. 2019,39(2):428-42.

19. Bronisz A, Rooj A, Krawczyński K, Peruzzi P, Salińska E, Nakano I, et al. The nuclear DICER-circular RNA complex drives the deregulation of the glioblastoma cell microRNAome. 2020,6(51).

20. Guo X, Qiu W, Liu Q, Qian M, Wang S, Zhang Z, et al. Immunosuppressive effects of hypoxia-induced glioma exosomes through myeloid-derived suppressor cells via the miR-10a/Rora and miR-21/Pten Pathways. Oncogene. 2018,37(31):4239-59.

21. Zhang X, Yuan S, Li H, Zhan J, Wang F, Fan J, et al. The double face of miR-320: cardiomyocytesderived miR-320 deteriorated while fibroblasts-derived miR-320 protected against heart failure induced by transverse aortic constriction. 2021,6(1):69.

22. Tustison N, Avants B, Cook P, Zheng Y, Egan A, Yushkevich P, et al. N4ITK: improved N3 bias correction. 2010,29(6):1310-20.

23. Shinohara R, Sweeney E, Goldsmith J, Shiee N, Mateen F, Calabresi P, et al. Statistical normalization techniques for magnetic resonance imaging. 2014,6:9-19.

24. Miller A, Shah R, Pentsova E, Pourmaleki M, Briggs S, Distefano N, et al. Tracking tumour evolution in glioma through liquid biopsies of cerebrospinal fluid. 2019,565(7741):654-8.

25. Zhang H, Deng T, Liu R, Bai M, Zhou L, Wang X, et al. Exosome-delivered EGFR regulates liver microenvironment to promote gastric cancer liver metastasis. 2017,8:15016.

26. Noman MZ, Janji B, Hu S, Wu JC, Martelli F, Bronte V, et al. Tumor-Promoting Effects of MyeloidDerived Suppressor Cells Are Potentiated by Hypoxia-Induced Expression of miR-210. Cancer Res. 2015,75(18):3771-87.

27. Chen C, Luo Y, He W, Zhao Y, Kong Y, Liu H, et al. Exosomal long noncoding RNA LNMAT2 promotes lymphatic metastasis in bladder cancer. J Clin Invest. 2020,130(1):404-21.

28. Hu B, Yang YT, Huang Y, Zhu Y, Lu ZJ. POSTAR: a platform for exploring post-transcriptional regulation coordinated by RNA-binding proteins. Nucleic Acids Res. 2017,45(D1):D104-D14.

29. Li J-H, Liu S, Zhou H, Qu L-H, Yang J-H. starBase v2.0: decoding miRNA-ceRNA, miRNA-ncRNA and protein-RNA interaction networks from large-scale CLIP-Seq data. Nucleic Acids Research. 2014,42(D1):D92-D7.

30. Wong N, Wang X. miRDB: an online resource for microRNA target prediction and functional annotations. Nucleic Acids Research. 2015,43(D1):D146-D52. 
31. Eso Y, Takai A, Matsumoto T, Inuzuka T, Horie T, Ono K, et al. MSH2 Dysregulation Is Triggered by Proinflammatory Cytokine Stimulation and Is Associated with Liver Cancer Development. Cancer Res. 2016,76(15):4383-93.

32. Wang LQ, Yu P, Li B, Guo YH, Liang ZR, Zheng LL, et al. miR-372 and miR-373 enhance the stemness of colorectal cancer cells by repressing differentiation signaling pathways. Molecular Oncology. 2018,12(11):1949-64.

33. Quail DF, Joyce JA. The Microenvironmental Landscape of Brain Tumors. Cancer Cell. 2017,31(3):326-41.

34. Meng W, Hao Y, He C, Li L, Zhu GJMc. Exosome-orchestrated hypoxic tumor microenvironment. 2019,18(1):57.

35. Cao L, Ren Y, Guo X, Wang L, Zhang Q, Li X, et al. Downregulation of SETD7 promotes migration and invasion of lung cancer cells via JAK2/STAT3 pathway. Int J Mol Med. 2020,45(5):1616-26.

36. Chen Y, Yang S, Hu J, Yu C, He M, Cai Z. Increased Expression of SETD7 Promotes Cell Proliferation by Regulating Cell Cycle and Indicates Poor Prognosis in Hepatocellular Carcinoma. PLoS One. 2016,11(5):e0154939.

37. Guo T, Wen XZ, Li ZY, Han HB, Zhang CG, Bai YH, et al. ISL1 predicts poor outcomes for patients with gastric cancer and drives tumor progression through binding to the ZEB1 promoter together with SETD7. Cell Death Dis. 2019,10(2):33.

38. Duan B, Bai J, Qiu J, Wang J, Tong C, Wang X, et al. Histone-lysine N-methyltransferase SETD7 is a potential serum biomarker for colorectal cancer patients. EBioMedicine. 2018,37:134-43.

39. Hoxhaj G, Manning BD. The PI3K-AKT network at the interface of oncogenic signalling and cancer metabolism. Nat Rev Cancer. 2020,20(2):74-88.

40. Porta C, Consonni FM, Morlacchi S, Sangaletti S, Bleve A, Totaro MG, et al. Tumor-Derived Prostaglandin E2 Promotes p50 NF-kappaB-Dependent Differentiation of Monocytic MDSCs. Cancer Res. 2020,80(13):2874-88.

41. Shurtleff MJ, Temoche-Diaz MM, Karfilis KV, Ri S, Schekman R. Y-box protein 1 is required to sort microRNAs into exosomes in cells and in a cell-free reaction. eLife. 2016,5.

\section{Figures}


a

Whole Transcriptome

Sequencing $(n=59)$

CSF-exosome Whole

Transcriptome Sequencing $(n=44) \geq$ twice

CSF-exosome Whole

Transcriptome Sequencing

b $(n=21) \geq$ thrice

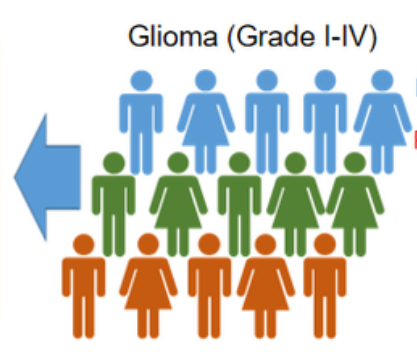

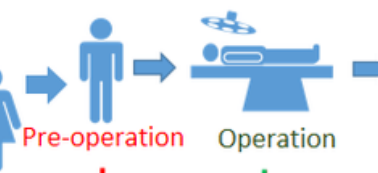

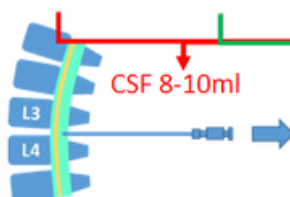

Lumbar Puncture

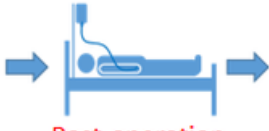

Post-operation 1

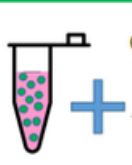

CSF-exosome

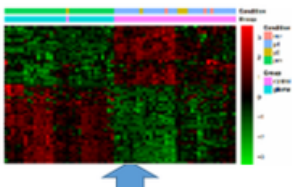

DNA WES (only tissue) mRNA Sequencing IncRNA Sequencing circRNA Sequencing Small RNA Sequencing (miRNA etc.)

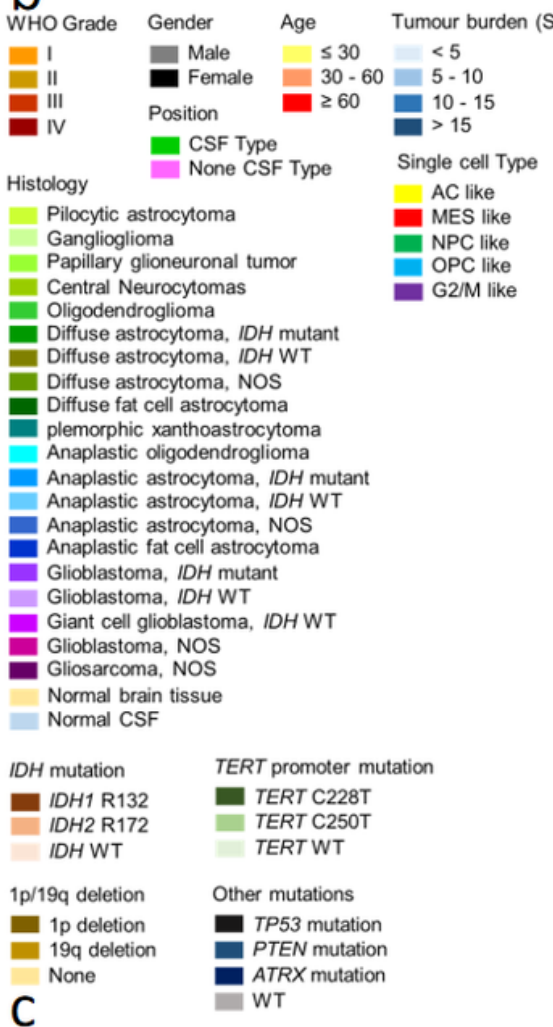

พพо Grase Histogry

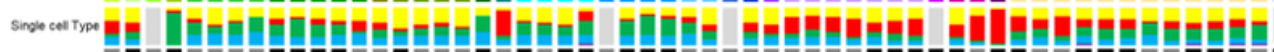
senser ${ }^{n} 90$ Position

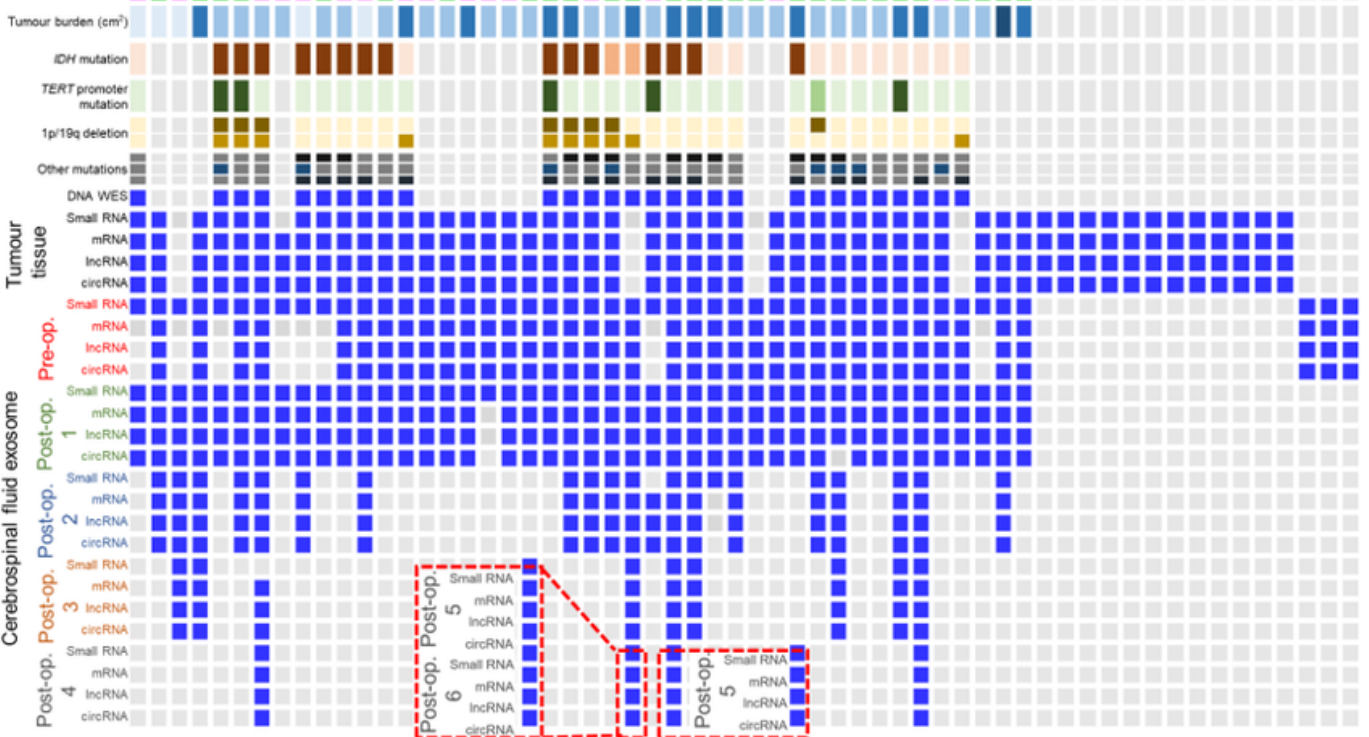

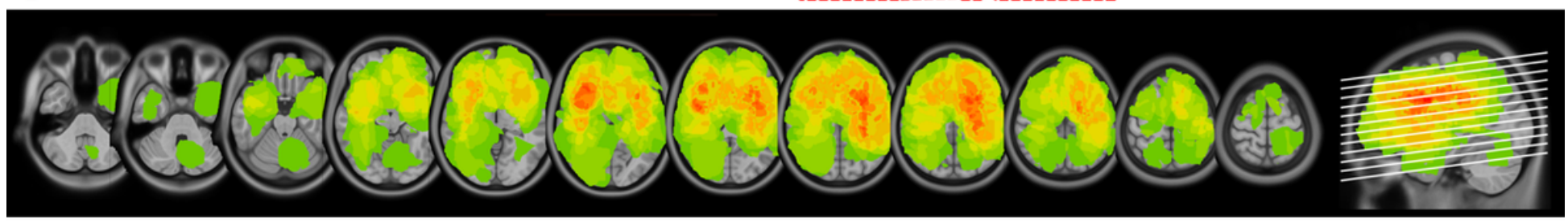

Figure 1

CSF sEV RNA database of glioma cohort. (a) Flowchart of our CSF sEV cohort study in 44 CSF sEV samples available patients with glioma from 148 WHO grade I to IV individuals. All 44 patients in this study were treated with surgery, and the tumor was totally removed under a surgical microscope. At the same time, multiple CSF specimens were obtained by a lumbar puncture at pre-operation and postoperation. Among them, the CSF specimens of 19 patients were further collected during the follow-up. These tumor specimens were undergoing whole exon and whole transcriptome sequencing, and the CSF samples were extracting sEV for following whole transcriptome sequencing. (b) Overview of the 44 glioma patients and 15 control with 12 normal brain tissues and three normal CSF of our cohort. Shown are the baseline characteristics, including WHO grade, histology, simulated single cell type, gender, age, 
position, tumor burden, and the most common genetic alterations. The blue squares below represent a panorama of all sequencing samples in the database. (c) The tumors' distribution locations in all 44 patients' brains were delineated by 3D Slicer (version 4.10.1).

a

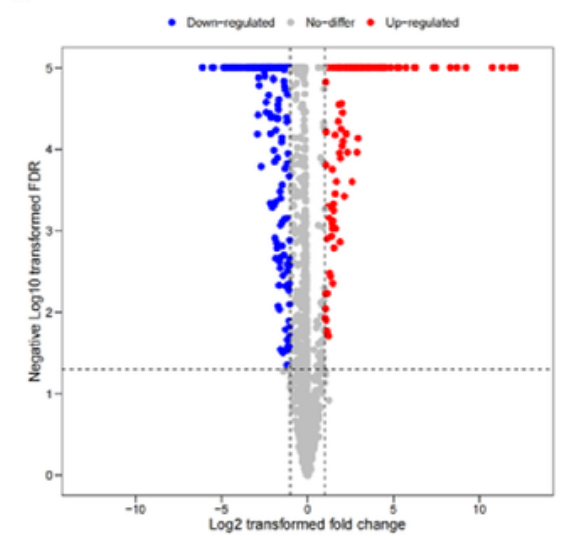

C

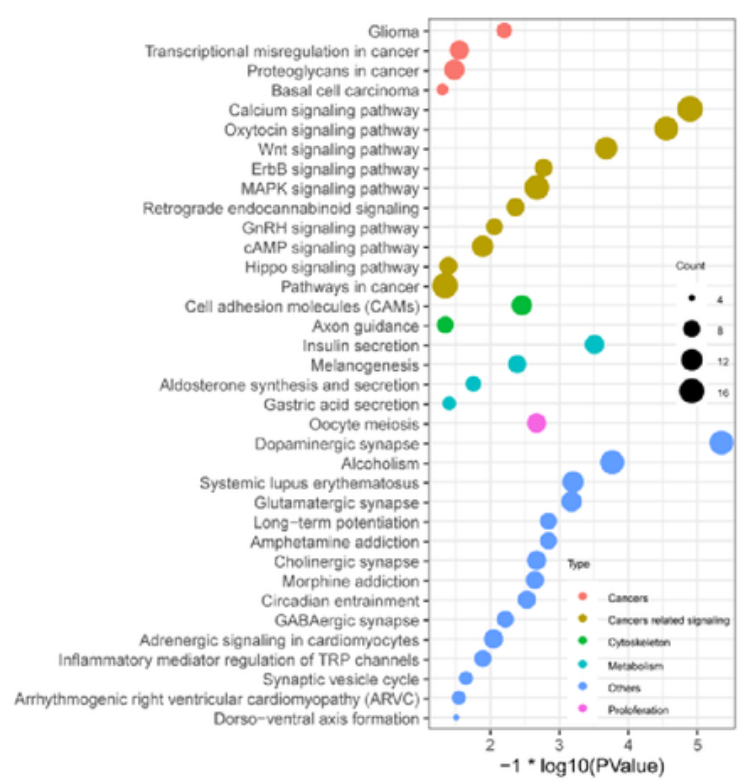

d

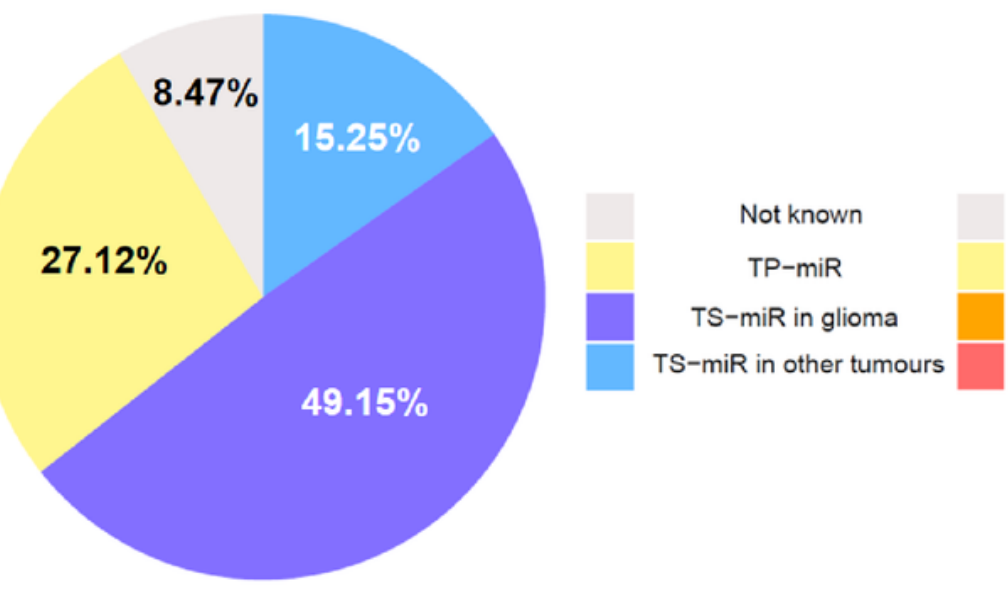

b
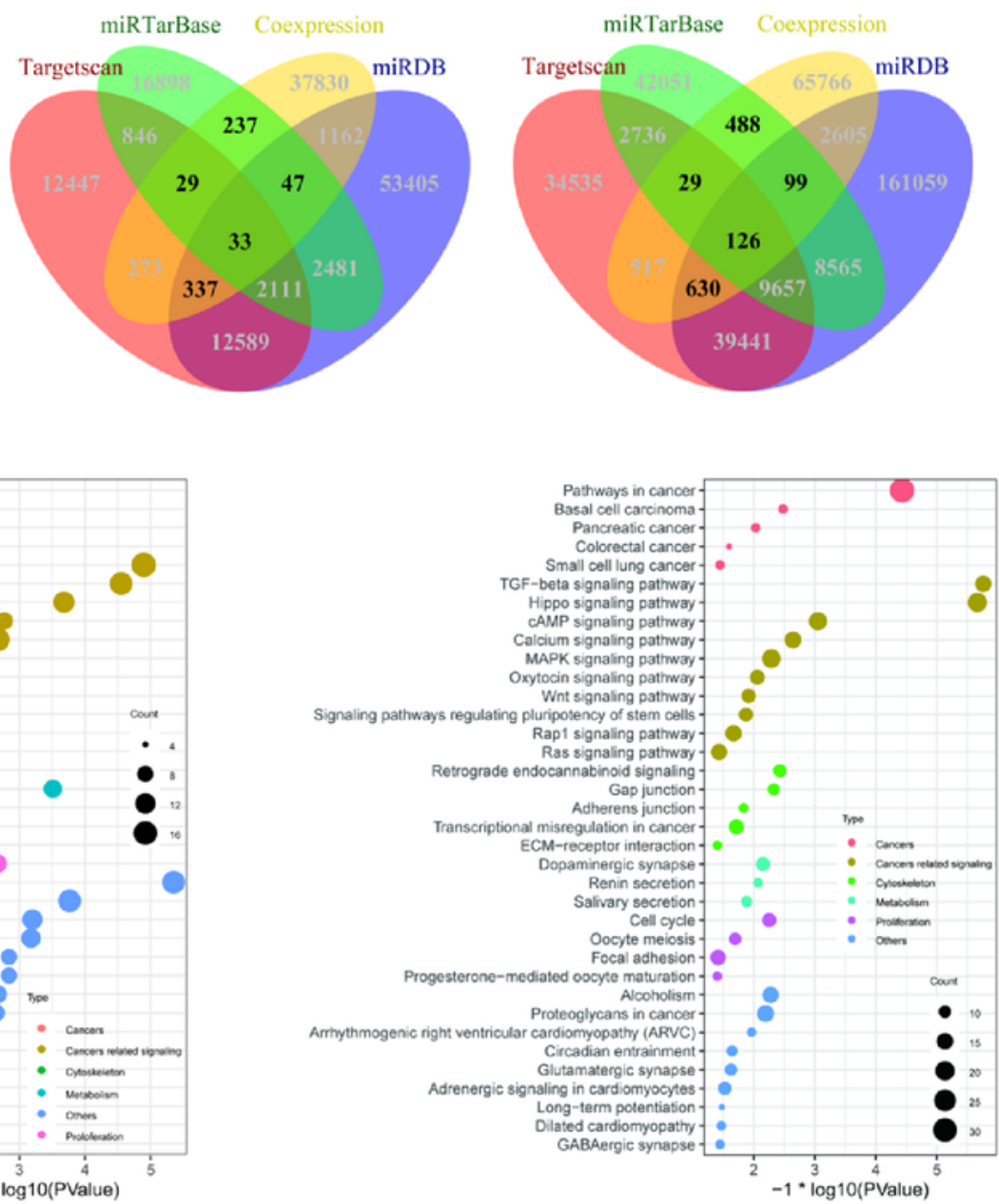

$7.14 \%$

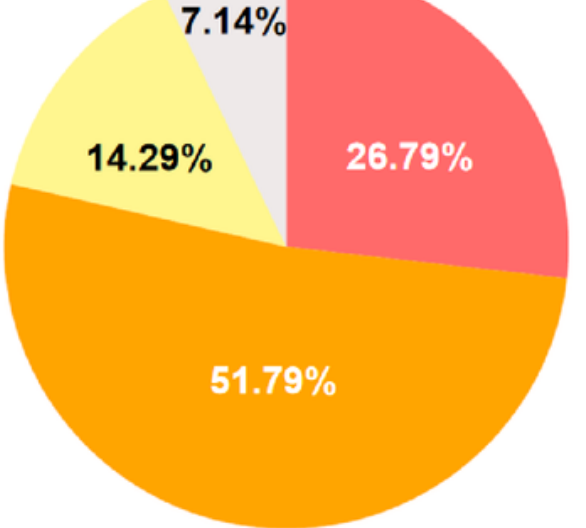

Figure 2

Tumor suppressive miRNAs dominated CSF sEV of glioma patients. (a) The volcano plot of differentially expressed miRNAs between pre-operation CSF sEV and glioma tissues. (b) Venn diagram of the predicted 
target mRNAs of above-mentioned differential expressed miRNAs (up- and down- regulated, respectively). The red, green, purple and yellow circle separately represented the targets obtained using Targetscan, miRTarBase, miRDB and Pearson Correlation Coefficient (PCC) analysis between the expression levels of mRNA and miRNA in glioma tissues (PCC $<-0.3$ and $p$ value $<0.05)$. The selected target sets shown in black font were retained for pathway enrichment analysis. (c) KEGG pathway enrichment analysis for targets of above-mentioned differential expressed miRNAs (up- and down- regulated bubble chart, respectively). (d) Pie chart of high-expressed CSF sEV miRNAs indicated the proportion of tumorsuppressive miRNAs (TS-miR) in glioma and other tumors, tumor-promoting miRNAs (TP-miR), and function unknown miRNA (up- and down- regulated, respectively). 
a

C

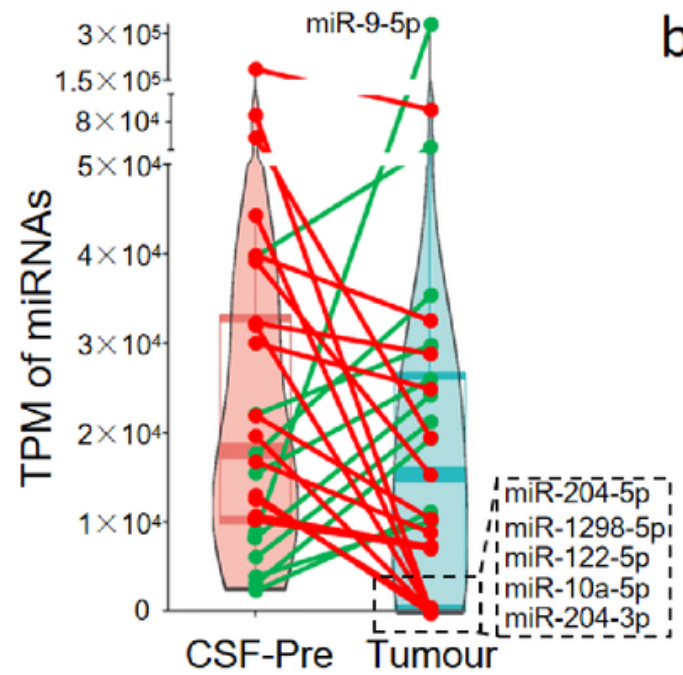

b

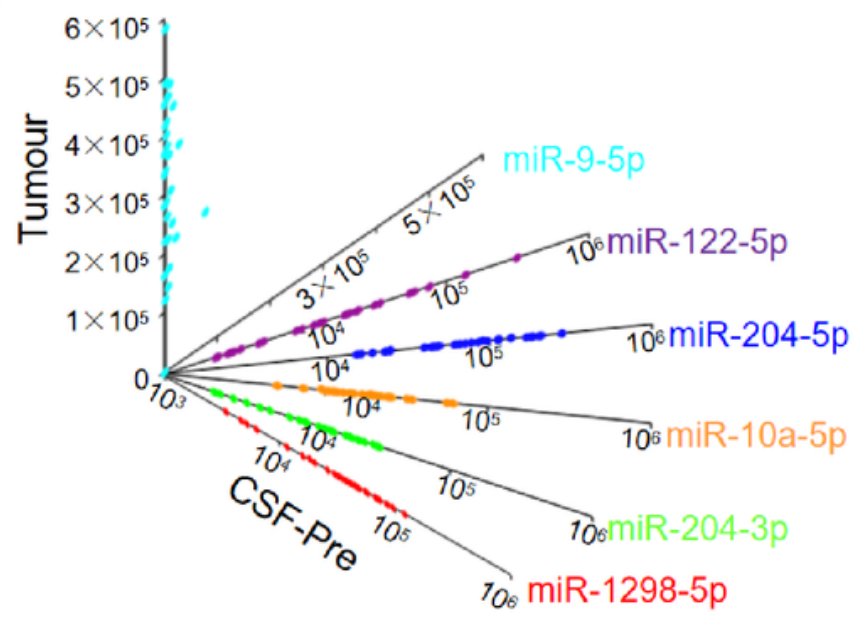

- cOR - HSE - LSE

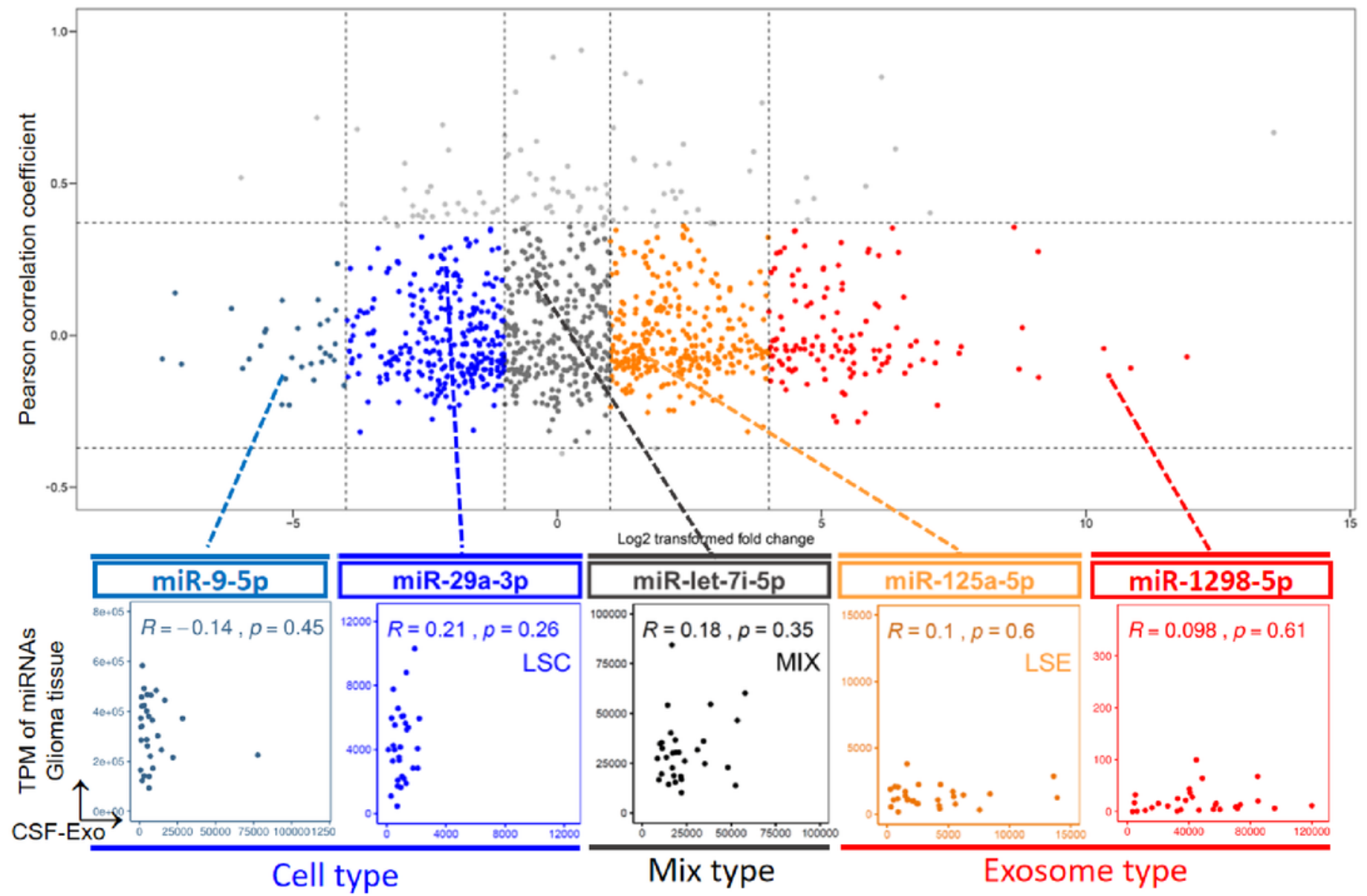

Figure 3

The CSF sEV miRNAs efflux (a) Violin-plot showing pair-wise comparison of high expressed miRNAs (TPM>10000) expression in pre-operation CSF sEV and glioma tissues. At left: each point represents the mean value of high expressed miRNAs in pre-operation CSF sEV. At right: each point represents the mean value of high expressed miRNAs in glioma tissues. Points of same miRNAs at left and right were link together. (b) A combination of scatter diagram of miR-9-5p, miR-122-5p, miRNA-204-5p, miR-10a-5p, miR- 
204-3p, miR-1298-5p. For differentially expressed miRNAs between tumor-burdened CSF sEV and sEV of non-tumorous CSF including post-operation No.1 and normal CSF, fold change and pair-wise Pearson Correlation Coefficient of miRNA expression between pre-operation CSF sEV and glioma tissues were calculated. miRNAs were divided into 5 types: high-selective sEV type (HSE), low-selective sEV type (LSE), mix type (MIX), low-selective cell type (LSC) and high-selective cell type (HSC) (fig 3c). For each type, a scatter diagram of representative miRNA is displayed.

a

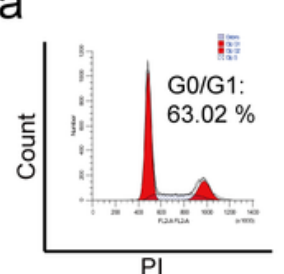

b

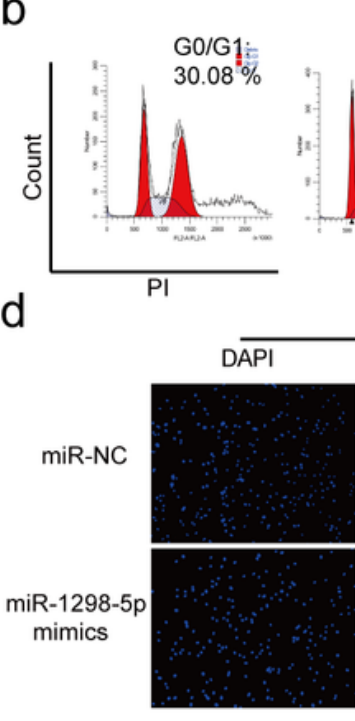

f

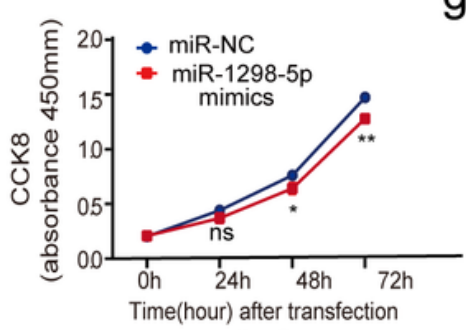

U87MG

i

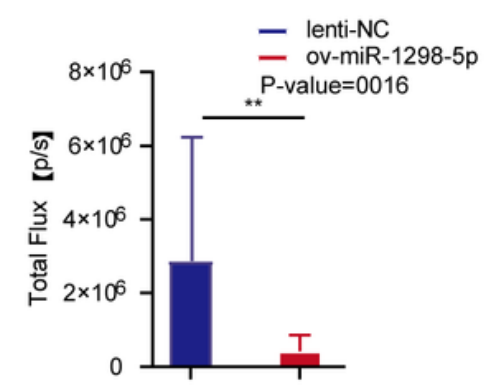

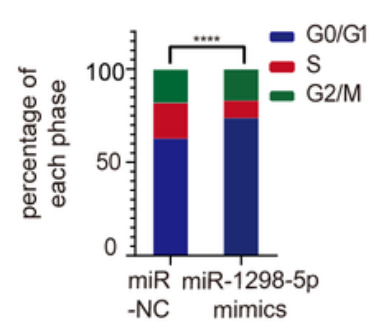

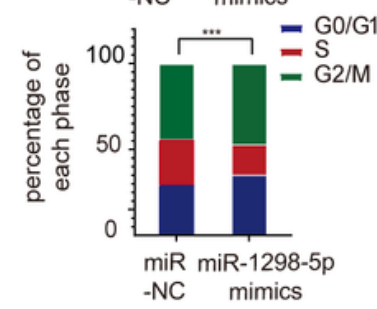

C
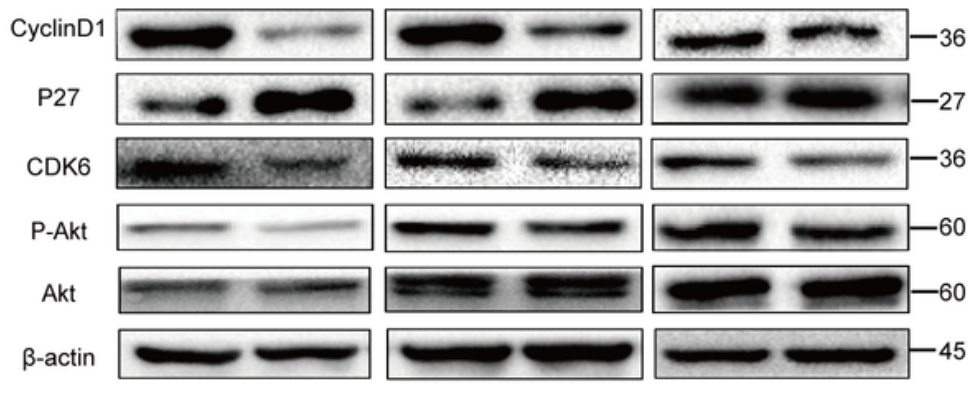

miR-NC miR-1298-5p miR-NC miR-1298-5p miR-NC miR-1298-5p Mr(KDa) mimics mimics mimics

U87MG

U251

e

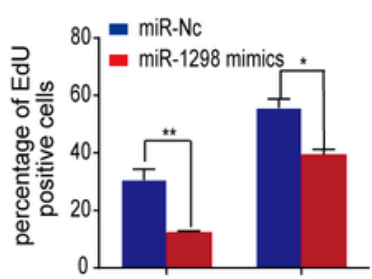

\section{Figure 4}


miR-1298-5p inhibited the proliferation of glioma in vitro and vivo $(a, b)$ Cell cycle analysis for U87MG and U251 cells transfected with miR-1298-5p mimics and a control sequence. The percentage of cells arrested in the G1/S phase is analyzed in a histogram (right panels). (c) The protein level of proliferation markers cyclinD1, P27, CDK6 and the AKT pathway in U87MG, U251 and P3 cells transfected with miR1298-5p mimics and a control sequence were assessed by western blotting. $\beta$-actin was used as the control for normalization. ( $\mathrm{d}-\mathrm{g})$ The proliferation capacity of U87MG and U251 cells transfected with miR1298-5p mimics were assessed using CCK8 assay and Edu assay. (scale bar, $100 \mu \mathrm{m}$ ) (h) In vivo bioluminescent imaging analysis of tumor growth in xenograft nude mice at day 10. (i) Quantification of luminescent signals in the Lenti-NC and OV-miR-1298-5p mice. (j) Survival analysis of nude mice orthotopically implanted with U87MG cells transfected with lentivirus overexpressing the control sequence or miR-1298-5p. ( $P=0.0180$ by log-rank analysis, data from 5 animals/group). ( $k$ ) H\&E staining of xenograft sections from miR-1298-5p overexpressing or negative control U87MG cell tissues on the same day of execution. Data are shown as the mean \pm SD of three independent experiments. Statistical significance was determined using one-way ANOVA test $\left(*, P<0.05,{ }^{*}, \mathrm{P}<0.01, * \star *, P<0.001\right)$. 

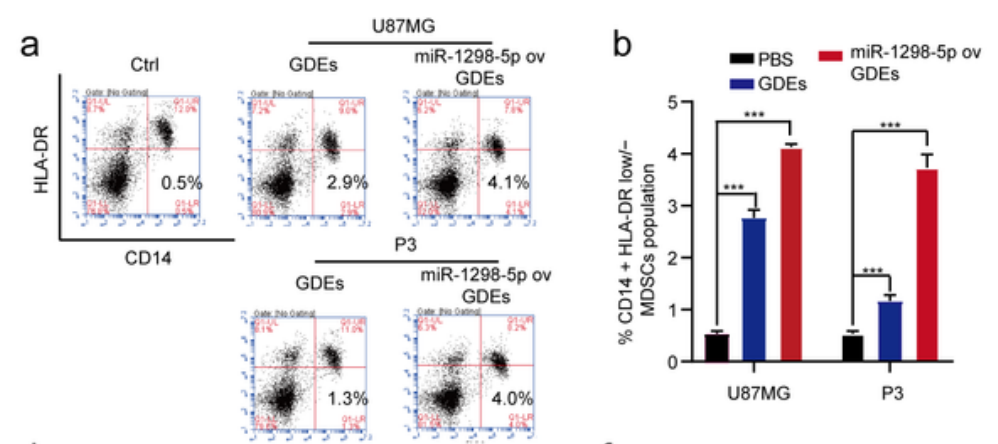

C
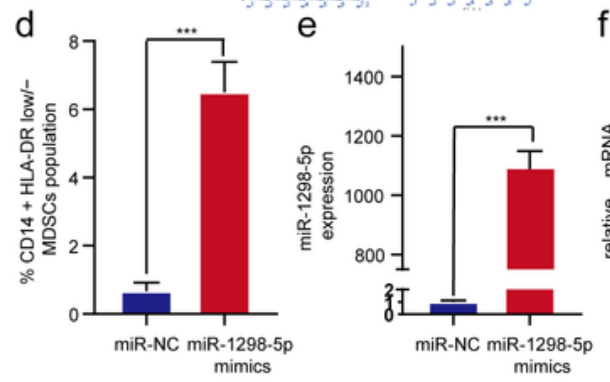

f $\quad$ g
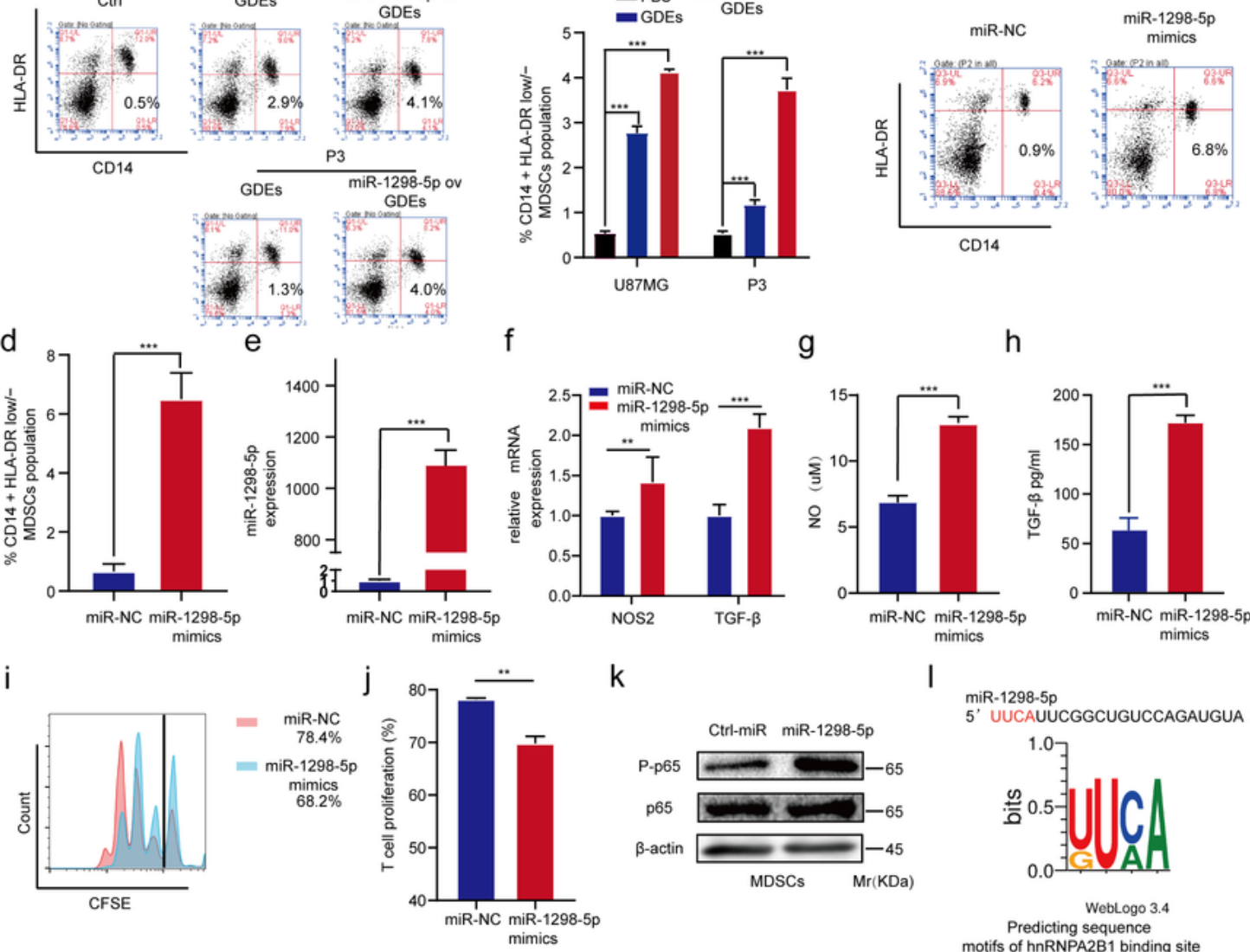

$\mathrm{k}$ I

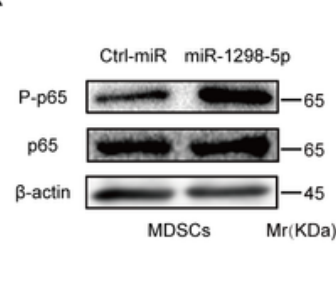

miR-1298-5p

5' UUCAUUCGGCUGUCCAGAUGUA 3 ,
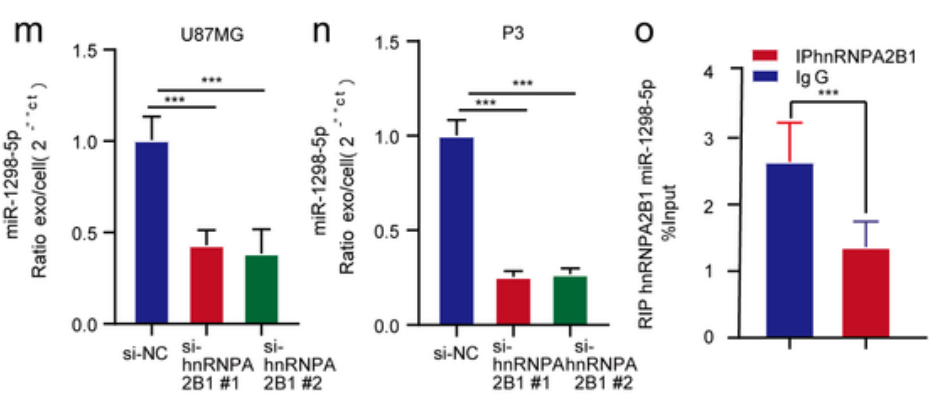

$\mathrm{p}$

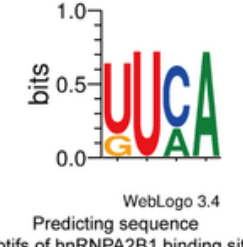

q

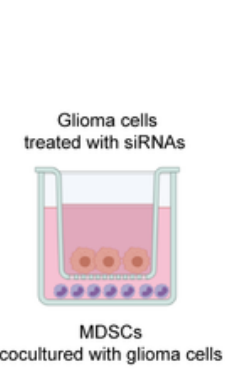

$r$

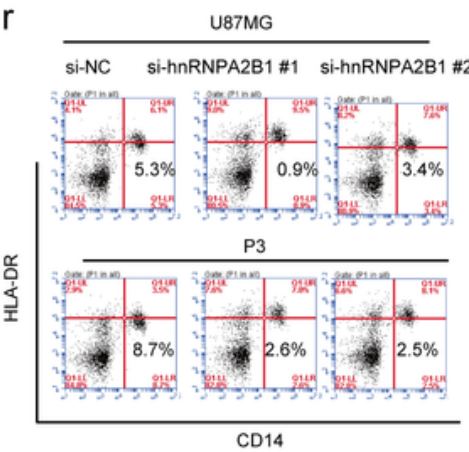

S

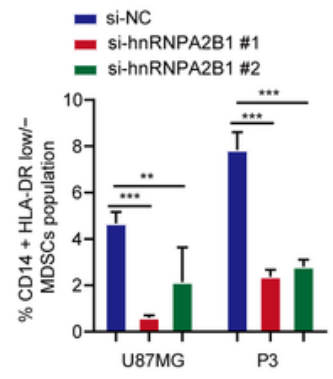

$\mathrm{t}$

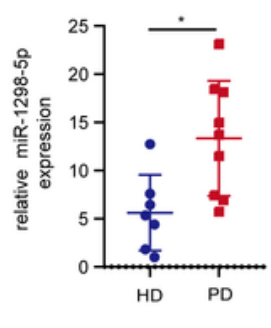

Figure 5

miR-1298-5p was sorted into exosomes via hnRNPA2B1 and promoted the immunosuppressive effects of MDSCs (a, b) Flow cytometry assay showed that GDEs overexpressing miR-1298-5p displayed a stronger MDSC induction ability than the PBS and GDEs. (c, d) Flow cytometry assay showed that miR-1298-5p upregulated the proportion of CD14+/HLA-DR low/-MDSCs population. (e, f) qRT-PCR demonstrated that miR-1298-5p increased the expression of NOS2 and TGF- $\beta$ in MDSCs. $(g, h)$ NO and TGF- $\beta$ in the 
supernatants of MDSCs were measured. $(i, j) C D 8+T$ cell proliferation was determined by flow cytometry three days later with CFSE dilution. $(k)$ The protein level of p65 and p-p65 in MDSCs transfected with miR1298-5p mimics and miR-NC were assessed by western blotting. $\beta$-actin was used as the control for normalization. (I) Sequence motifs of hnRNPA2B1 binding site predicted by POSTAR2. $(m, n)$ qRT-PCR assay analyzed the exo/cell ratio of miR-1298-5p in U87MG and P3 after knocking down hnRNPA2B1. (o) RIP analysis using the anti-hnRNPA2B1 antibody revealed that miR-1298-5p interacted with hnRNPA2B1 in U87MG cells. The negative control, IgG. (p) RNA pull-down and Western blot with U87MG lysate confirmed that miR-1298-5p was associated with hnRNPA2B1. (q, r, s) Cultured MDSCs with U87MG and P3 knocking down hnRNPA2B1 and analyzed the ratio of CD14+ HLA-DR low/- MDSCs population using Flow cytometry assay. $(\mathrm{t})$ The expression of miR-1298-5p in MDSCs of patents was higher than health donors. Data are shown as the mean \pm SD of three independent experiments. Statistical significance was determined using one-way ANOVA test $\left({ }^{*}, \mathrm{P}<0.05, * \star, \mathrm{P}<0.01\right.$, $\left.* \star \star, P<0.001\right)$. 

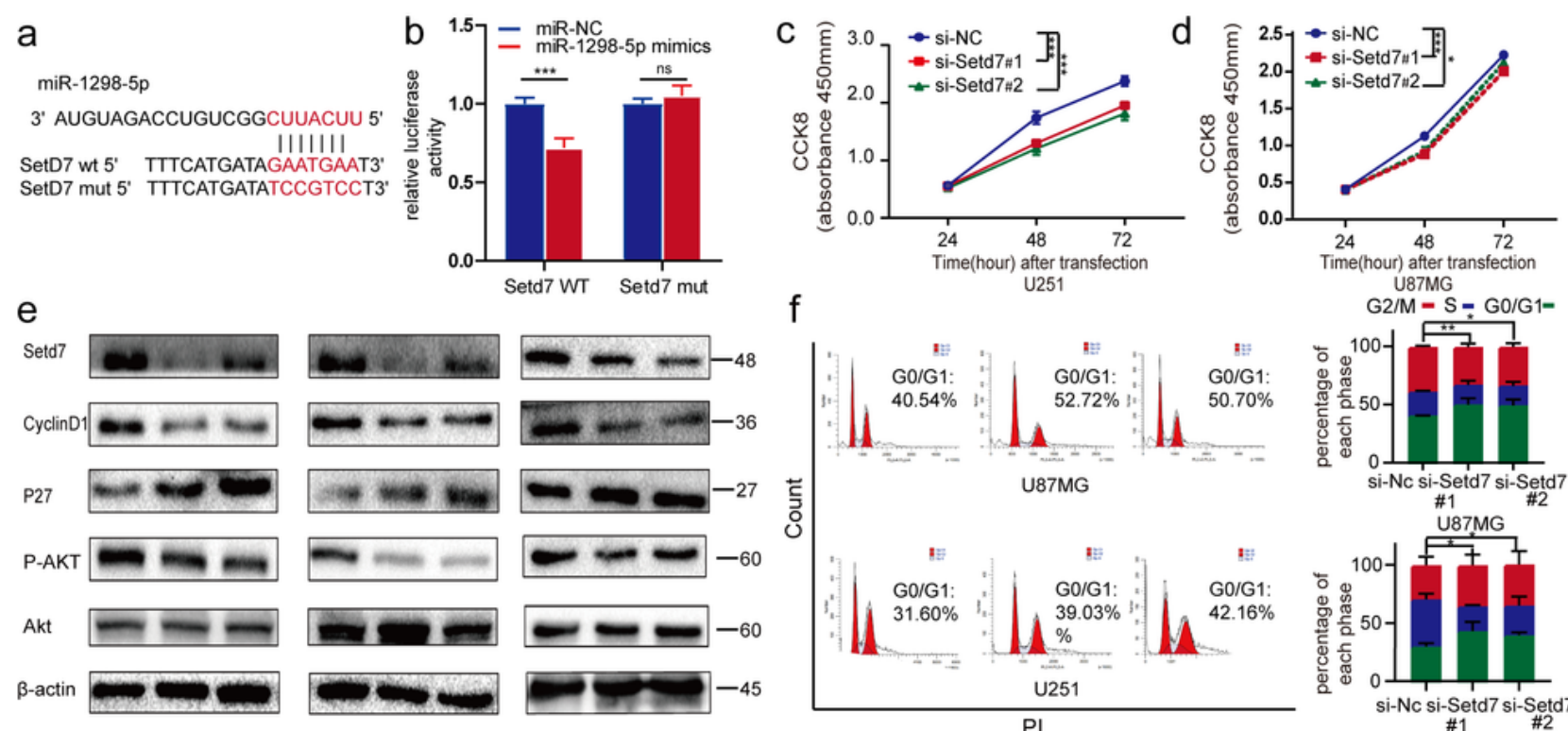

f
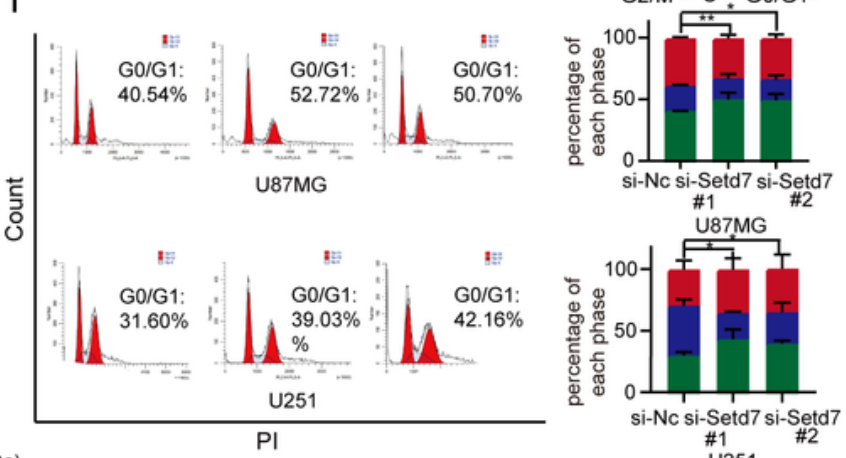

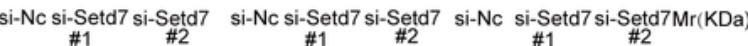
U87MG U251 P3
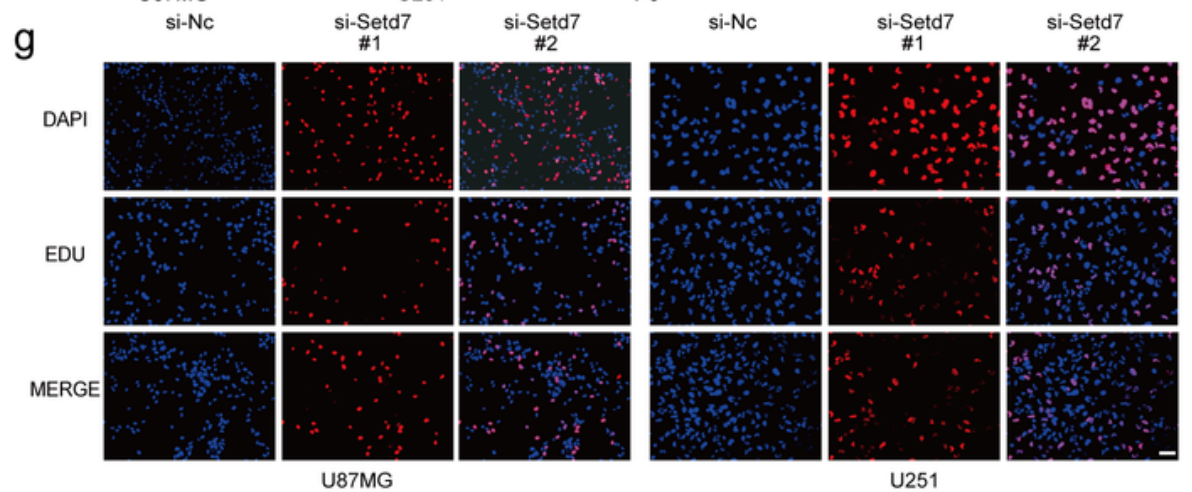

h
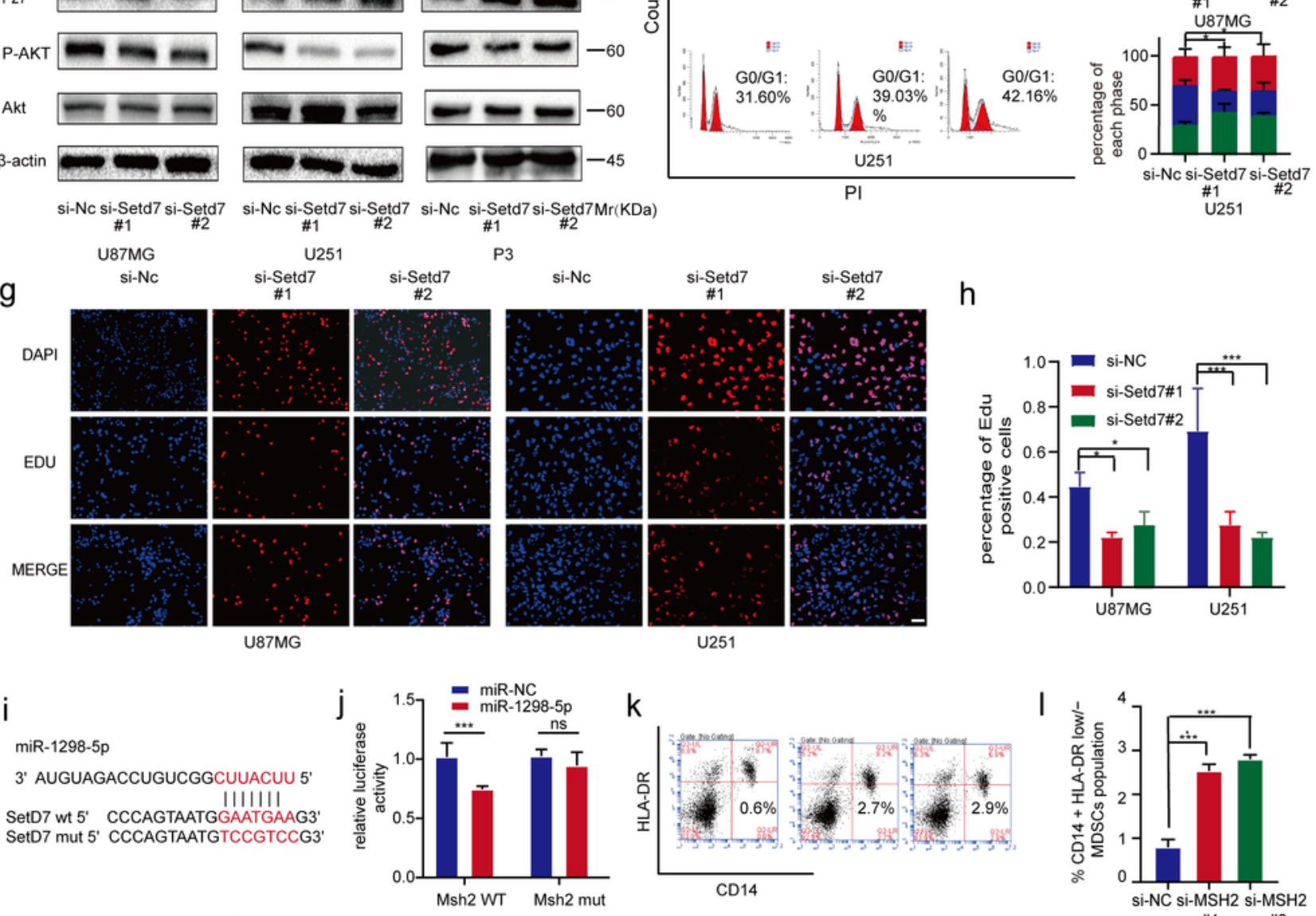

m

n

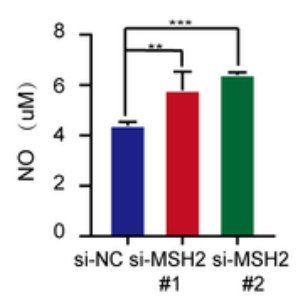

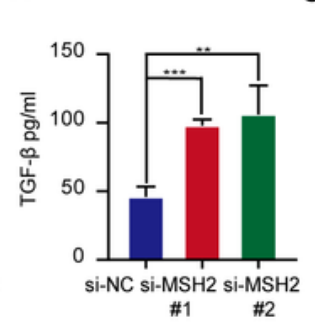

0
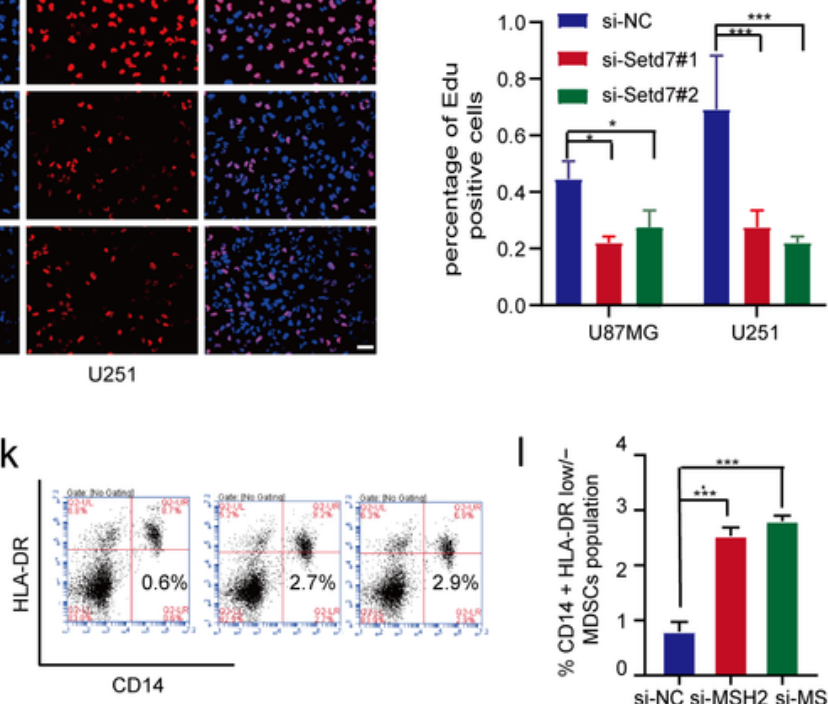

q
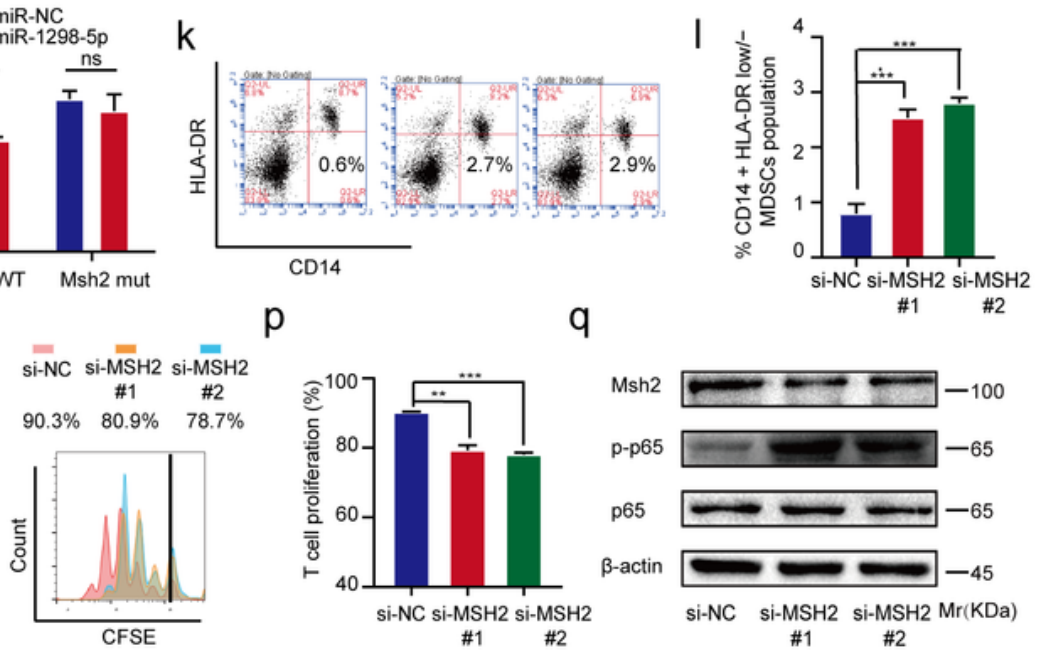

Figure 6

miR-1298-5p targeted SETD7 in glioma and MSH2 in MDSCs (a) Construction of wild type (WT) and mutant type (MUT) luciferase reporter vectors based on the predicted binding site of miR-1298-5p in SETD7. (b) 293T cells were co-transfected with the reporter vectors and miR-1298-5p or miR-Nc.

Luciferase activity was assessed $48 \mathrm{~h}$ after transfection. (c, d) The proliferation capacity of U87MG and U251 cells after SETD7 knockdown were assessed using CCK8 assay. (e) The protein level of SETD7, 
cyclinD1, P27, p-Akt, Akt in U87MG, U251 and P3 cells knocking down SETD7 were assessed by western blotting. $\beta$-actin was used as the control for normalization. (f) Cell cycle analysis for U87MG and U251 cells knocking down SETD7. The percentage of cells arrested in the G1/S phase is analyzed in a histogram (right panels). ( $\mathrm{g}, \mathrm{h}$ ) The proliferation capacity of U87MG and U251 cells after SETD7 knockdown were assessed using the Edu assay. (scale bar, $100 \mu \mathrm{m}$ ) (i) Construction of wild type (WT) and mutant type (MUT) luciferase reporter vectors based on the predicted binding site of miR-1298-5p in MSH2. (j) 293T cells were co-transfected with the reporter vectors and miR-1298-5p or miR-Nc. Luciferase activity was assessed $48 \mathrm{~h}$ after transfection. $(k, l)$ Flow cytometry assay showed that MSH2 knockdown upregulated the proportion of CD14+ HLA-DRlow/- MDSCs population. $(m, n) N O$ and TGF- $\beta$ in the supernatants of MDSCs were measured after MSH2 knockdown. (o, p) CD8+ T cell proliferation was determined by flow cytometry three days later with CFSE dilution. (q) The protein level of p65 and p-p65 in MDSCs transfected with MSH2 siRNAs were accessed by western blotting. $\beta$-actin was used as the control for normalization. Data are shown as the mean \pm SD of three independent experiments. Statistical significance was determined using one-way ANOVA test $\left(*, P<0.05,{ }^{*}, \mathrm{P}<0.01, * \star *, P<0.001\right)$. 
a
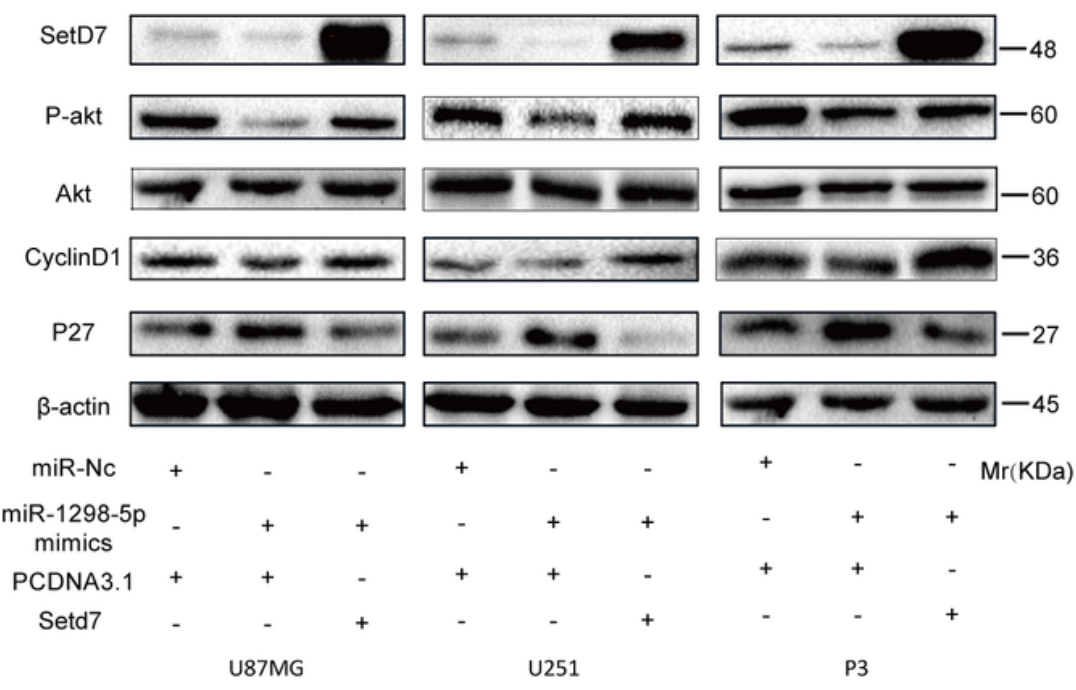

\section{5}

b
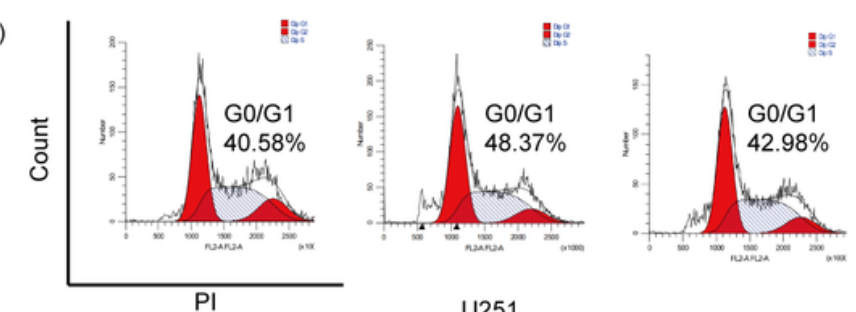

U251

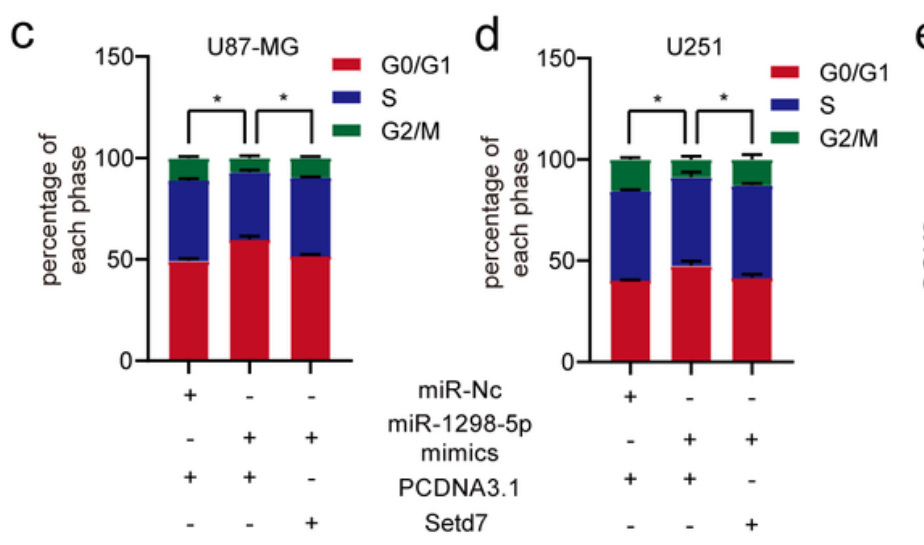

e

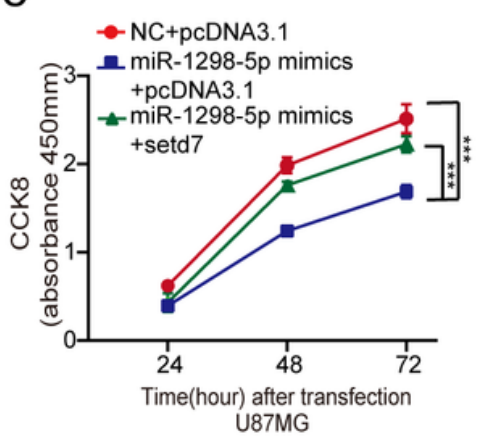

f
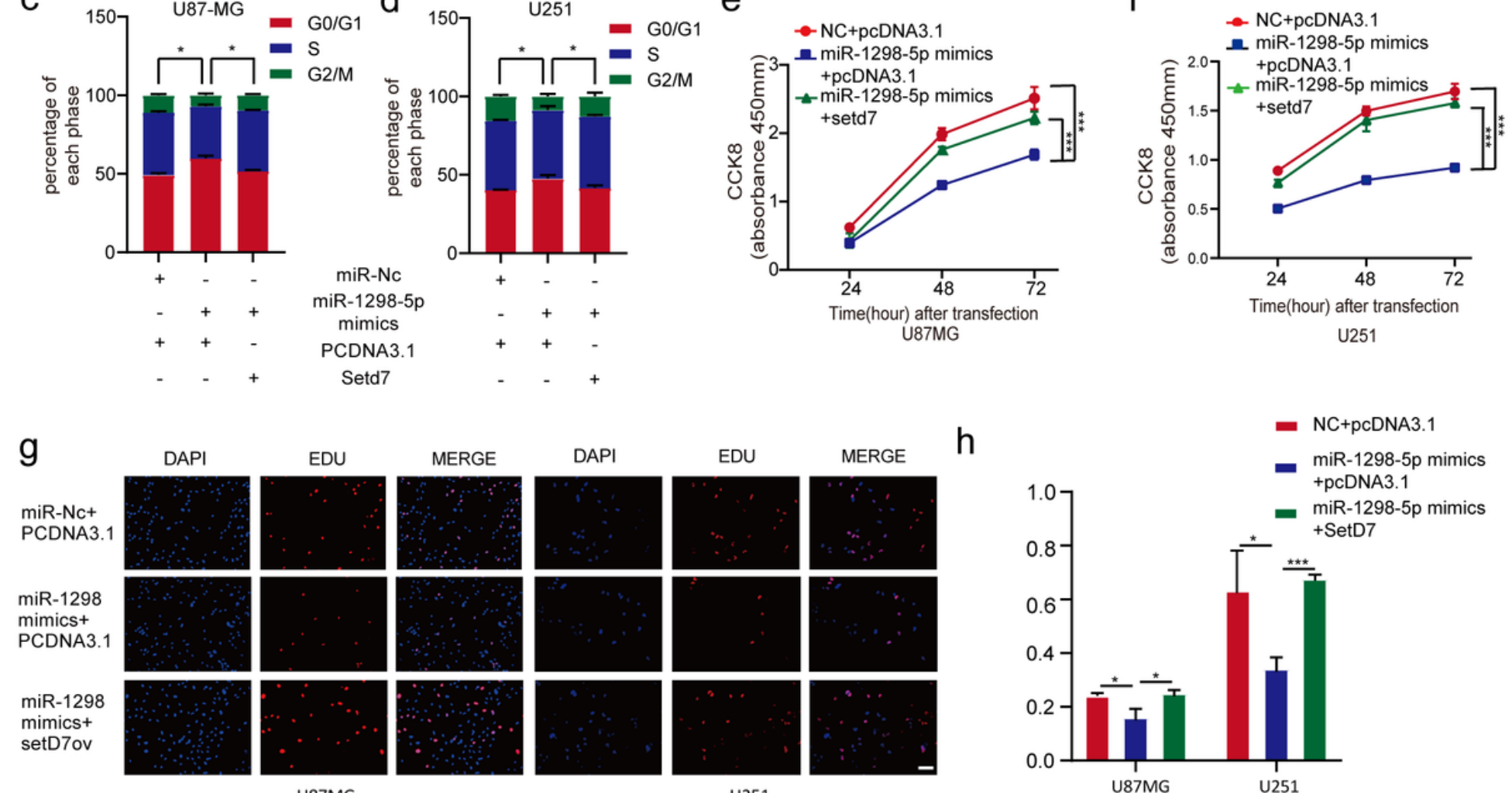

h

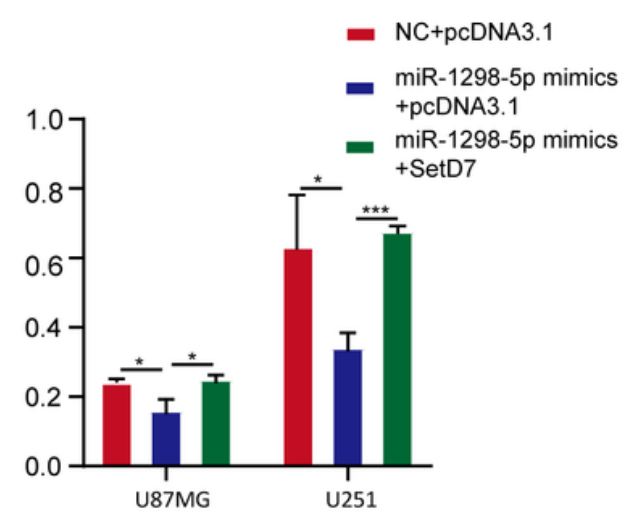

Figure 7

The effects of miR-1298-5p overexpression in glioma could be partially attenuated by SETD7 overexpression (a) Protein level of SETD7, cyclinD1, P27, p-Akt, Akt in U87MG, U251 and P3 cells transfected with miR-1298-5p mimics and pcDNA3.1-SETD7 or pcDNA3.1 were assessed by western blotting. $\beta$-actin was used as the control for normalization. (b-d) Cell cycle analysis for U87MG and U251 cells. The percentage of cells transfected with miR-1298-5p mimics arrested in the G1/S phase can be attenuated by SETD7 overexpression. (e-h) The proliferation capacity of U87MG and U251 cells treated 
as described above were assessed using CCK8 assay and EDU assay. (scale bar, $100 \mu$ m) Statistical significance was determined using one-way ANOVA test $(*, P<0.05$, **, $P<0.01$, ***, $P<0.001)$.
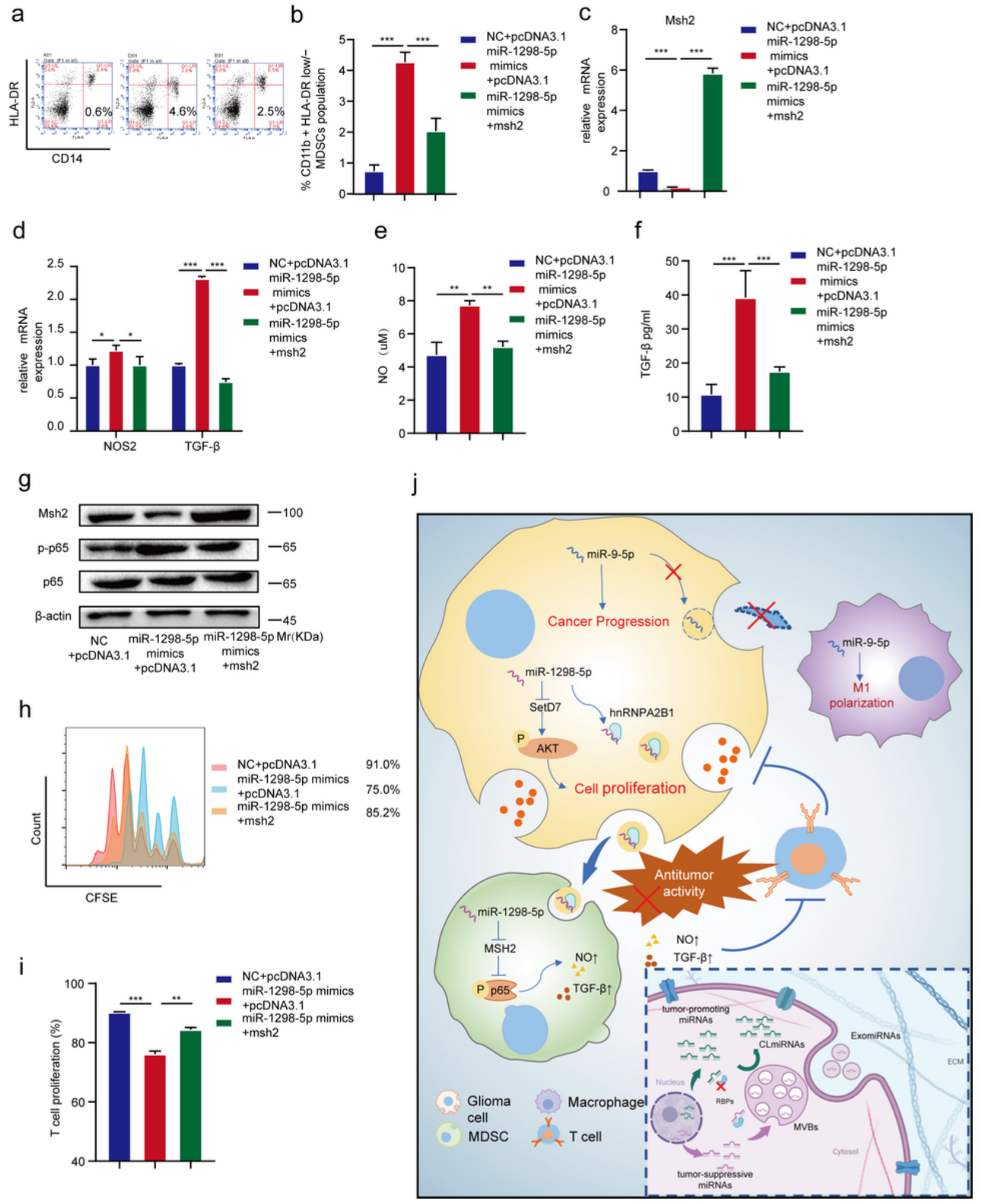

Figure 8

The effects of miR-1298-5p overexpression in MDSCs could be partially attenuated by MSH2 overexpression $(a, b)$ MDSCs transfected with miR-1298-5p mimics and pcDNA3.1-MSH2 or pcDNA3.1 were assessed by Flow cytometry assay. (c, d) The qRT-PCR assay showed the change of the expression 
of NOS2 and TGF- $\beta$ in MDSCs treated as described above. (e, $f)$ NO and TGF- $\beta$ in the supernatants of MDSCs treated as described above. (g) The protein level of MSH2, p-p65 and p65 in MDSCs treated as described above were assessed by western blotting. $\beta$-actin was used as the control for normalization. (h, i) CD8+ T cell proliferation was determined by flow cytometry three days later with CFSE dilution. (j) Schematic model showing that glioma selectively sorted oncosuppressor miR-1298-5p into exosomes and exosomal miR-1298-5p could promote the Immunosuppressive effects on of MDSCs. Moreover, miR$9-5 p$ could promote glioma progression and induce M1 polarization of macrophages. Therefore, miR-9-5p was trapped inside cells. Data are shown as the mean \pm SD of three independent experiments. Statistical significance was determined using one-way ANOVA test $(*, P<0.05, * \star, P<0.01$, ***, $P<0.001)$.

\section{Supplementary Files}

This is a list of supplementary files associated with this preprint. Click to download.

- supplementaryfigures.docx

- TableS1.docx

- Tables2.docx

- TableS3.xlsx

- TableS4.docx

- TableS5.xIsx

- TableS6.docx 